\title{
Review Article \\ Surface Potential Analysis of Nanoscale Biomaterials and Devices Using Kelvin Probe Force Microscopy
}

\author{
Hyungbeen Lee, ${ }^{1}$ Wonseok Lee, ${ }^{1}$ Jeong Hoon Lee, ${ }^{2}$ and Dae Sung Yoon ${ }^{3}$ \\ ${ }^{1}$ Department of Biomedical Engineering, Yonsei University, Wonju 26493, Republic of Korea \\ ${ }^{2}$ Department of Electrical Engineering, Kwangwoon University, Seoul 139-701, Republic of Korea \\ ${ }^{3}$ Department of Bio-Convergence Engineering, Korea University, Seoul 02841, Republic of Korea \\ Correspondence should be addressed to Jeong Hoon Lee; jhlee@kw.ac.kr and Dae Sung Yoon; dsyoon@korea.ac.kr
}

Received 18 November 2015; Accepted 29 December 2015

Academic Editor: Meng Sun

Copyright (C) 2016 Hyungbeen Lee et al. This is an open access article distributed under the Creative Commons Attribution License, which permits unrestricted use, distribution, and reproduction in any medium, provided the original work is properly cited.

\begin{abstract}
In recent years, Kelvin probe force microscopy (KPFM) has emerged as a versatile toolkit for exploring electrical properties on a broad range of nanobiomaterials and molecules. An analysis using KPFM can provide valuable sample information including surface potential and work function of a certain material. Accordingly, KPFM has been widely used in the areas of material science, electronics, and biomedical science. In this review, we will briefly explain the setup of KPFM and its measuring principle and then survey representative results of various KPFM applications ranging from material analysis to device analysis. Finally, we will discuss some possibilities of KPFM on whether it is applicable to various sensor systems. Our perspective shed unique light on how KPFM can be used as a biosensor as well as equipment to measure electrical properties of materials and to recognize various molecular interactions.
\end{abstract}

\section{Introduction}

Kelvin probe force microscopy (KPFM) is a success story of atomic force microscopy (AFM) being combined with the Kelvin probe method. KPFM was invented in 1991 [1] and it has been now widely used to measure work functions of various materials or nanoscale devices and to observe surface charge distributions of them at atomic or molecular scales [2-5]. Compared to the conventional Kelvin probe, KPFM has the biggest advantage of its high spatial resolution [6-8], which is brought about because it uses a conducting cantilevered beam with a sharp tip. Over the past decades, because of its advantage, KPFM became a unique tool for measuring the surface charge distribution or the work functions of metal/semiconductor surfaces [9-13] and semiconductor devices $[14,15]$.

KPFM is able to obtain the work function of sample by measuring contact potential difference (CPD) between the sample surface and the conducting cantilever tip, where the CPD is the difference between the work functions of the sample surface and the tip. The CPD between two materials depends on a variety of their electronic/electrical parameters. Measurement of the CPD can be used not only for measuring work functions of metal/semiconductor surfaces but also for mapping surface potentials of biomolecules/macromolecules (e.g., proteins [16], single DNA molecules [17, 18], and amyloid fibrils $[19,20])$. In general, surface potential is known to represent surface charge of various samples via electrostatic potential. With this principle, KPFM may be suitably adopted to investigate molecular interactions that lack topological differences but have distinguishable differences in surface potential. Furthermore, the capability for discriminating the difference in surface potential between before and after bioassay allows one to utilize KPFM for developing a diagnostic platform based on testing biomolecular interactions.

Some researchers are now trying to develop a new nano/biodiagnostic platform based on a combination of KPFM and nanoparticles or nanodevices [21]. Because the KPFM technique is basically a scanning probe method, it has certain unique advantages as a diagnostic system, including (i) direct detection of molecules by mapping surface potential, (ii) label-free method, and (iii) detection of single 
molecules with high spatial resolution. These advantages represent that a KPFM-based nano/biodiagnostic system would be effective for performing bioassays not being able to be accomplished by other systems.

This review article presents the state of the art in KPFM applications to surface analysis of nanomaterials or bioassays using nano/biosensing devices. Before we describe KPFM applications in depth, we will briefly introduce the principle of KPFM and explain how the surface potential of the sample is obtained. Of KPFM applications, we will discuss three categories. The first is the characterization of electrical properties in various nanomaterials. We explore some examples of surface potential measurements of various nanoparticles, two-dimensional (2D) layered nanomaterials, and even single-layered nanomaterials. The second category is KPFM imaging of various biomolecules including DNA and proteins. It will be shown that KPFM can be used not only to measure the surface potential of a single biomolecule itself but also to monitor the molecular interaction with surrounding molecules. The last category is biosensor or diagnostic system applications of KPFM. In this section, we will demonstrate that KPFM has a huge potential as a new biosensor platform with ultrasensitivity, providing some examples of the KPFM based biosensors. Finally, we will discuss the perspective and various possibilities of KPFM.

\section{Principle of Kelvin Probe Force Microscopy}

2.1. Fundamental of KPFM. KPFM can measure the electrostatic surface potential of a sample at the micro- to nanoscales. Hardware of KPFM is based on a general AFM system and the measurement of the electrostatic surface potential is obtained by detecting the motion of a conducting cantilever that is caused by an electrostatic force between a tip mounted on the cantilever and the sample. The measured electrostatic force represents the CPD between the sample surface and the conducting cantilever tip. When a metallic material is electrically isolated from other materials, it has an inherent Fermi level due to its microstructure, composition, and work function. However, when two metallic materials are in contact electrically, the materials are in thermodynamic equilibrium with each other and have equal Fermi levels. If two materials are different (have different work functions), there exists a CPD between them when kept in contact. A common method for determining the CPD is known as Kelvin method [22] (it is why KPFM adopts the term Kelvin). With the Kelvin method, two plates (i.e., a vibrating reference electrode on a cantilevered beam and a sample) are aligned for forming a capacitor, and a dc bias is applied between them. By the vibrating reference electrode, an electric current $i(t)$ is generated, as shown in Figure 1(a). With $\omega$ being the frequency of vibration, the generated current is given by

$$
i(t)=V_{\mathrm{CPD}} \omega \Delta C \cos \omega t,
$$

where $\Delta C$ is variation of capacitance and $V_{\mathrm{CPD}}$ is contact potential between two plates. The $V_{\mathrm{CPD}}$ is simply defined as

$$
V_{\mathrm{CPD}}=\frac{\phi_{1}-\phi_{2}}{-e}
$$

where $\phi_{1}$ and $\phi_{2}$ are work functions of two conductors (with $\mathrm{KPFM}, \phi_{1}=\phi_{\text {tip }}$ and $\phi_{2}=\phi_{\text {sample }}$ ), and $e$ is electrical charge of an electron. The $V_{\mathrm{CPD}}$ is obtained when the $V_{\mathrm{DC}}$ is varied until the current $i(t)$ is minimized.

KPFM system also adopts a similar mechanism to the Kelvin method. When a conducting cantilever tip approaches the sample, an electrostatic force is caused by the difference between their Fermi levels. An energy diagram representing two Fermi levels is shown in Figure 1(b). However, when a conducting cantilever tip and the sample are in contact electrically, both Fermi levels are rearranged through the interchange of electrons. By this electron movement, the tip and the sample reach thermodynamic equilibrium and this state is shown in Figure 1(d).

Figure 2 depicts a block diagram explaining how a general KPFM system operates. An AC voltage $\left(V_{\mathrm{AC}}\right)$ with a dc offset bias $\left(V_{\mathrm{DC}}\right)$ is applied between a conducting cantilever tip and a sample, which results in generation of an electrostatic force via the $V_{\mathrm{CPD}}$. The electrostatic force causes the deflection of the conducting cantilever and it is monitored by a laser and a PSD (position sensitive detector). The frequency component $\left(f_{\mathrm{AC}}\right)$ is extracted from the obtained PSD signal through a lock-in amplifier. A feedback controller applies the $V_{\mathrm{DC}}$ to the AFM system until the current $f_{\mathrm{AC}}$ component is minimized. Consequently, the $V_{\mathrm{CPD}}$ is obtained by adjusting the $V_{\mathrm{DC}}$ component.

2.2. Two Different Modes of KPFM. KPFM is driven by a variety of working modes, depending on manufacturers or brands. Regardless of their subtle difference in operation, most of scientists classify them into two different modes (i.e., lift mode and dual-frequency mode) [2]. Lift-mode KPFM, also known as amplitude modulated KPFM (AMKPFM), is based on a dual line-scan system. In the first scan of cantilever, surface topography is obtained by standard tapping mode. In the second scan, the cantilever ascends to lift scan height and traces the previously recorded surface topography to detect the surface potential of sample. However, dual-frequency mode KPFM, also known as frequency modulated KPFM (FM-KPFM), is based on a single-scan system. Dual-frequency mode KPFM applies an AC signal to the cantilever at low frequency $\left(\omega_{m}\right)$, while the cantilever is mechanically excited nearly at its resonance frequency $\left(\omega_{\text {res }}\right)$. The applied AC signal at low frequency induces a modulation of electrostatic force, which is detected by the superimposed oscillation $\left(\omega_{\text {res }} \pm \omega_{m}\right)$ in the amplitude versus frequency plot representing the mechanical oscillation of cantilever, leading to separation of the topography $\left(\omega_{\text {res }}\right)$ and the surface potential signal $\left(\omega_{\text {res }} \pm \omega_{m}\right)$ of sample. Dualfrequency mode KPFM has higher spatial resolution than lift-mode KPFM [23], because dual-frequency mode KPFM detects the surface potential signal more directly than liftmode KPFM. Nevertheless, there are some researchers that prefer lift-mode KPFM to dual-frequency mode because of its high signal-to-noise (SN) ratio. It is thought that high $\mathrm{SN}$ ratio in lift mode will help one to perform precise measurement of surface potentials [19, 20, 24-27]. 


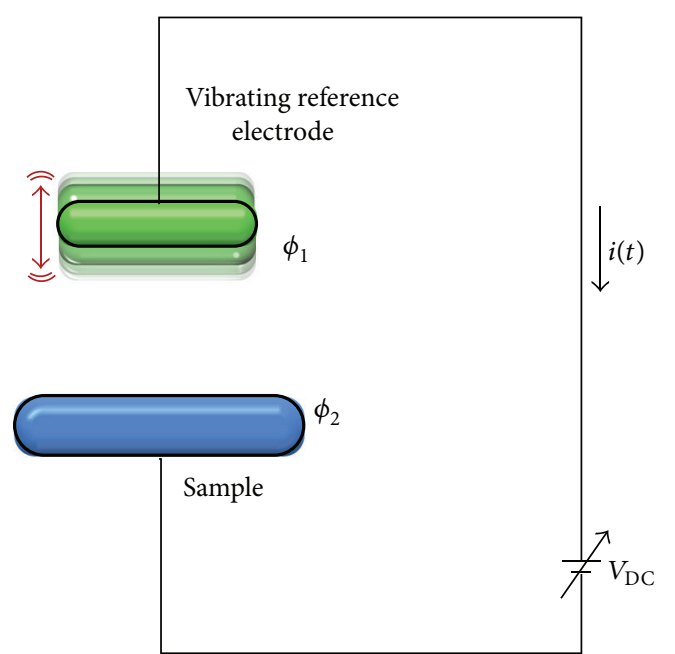

(a)

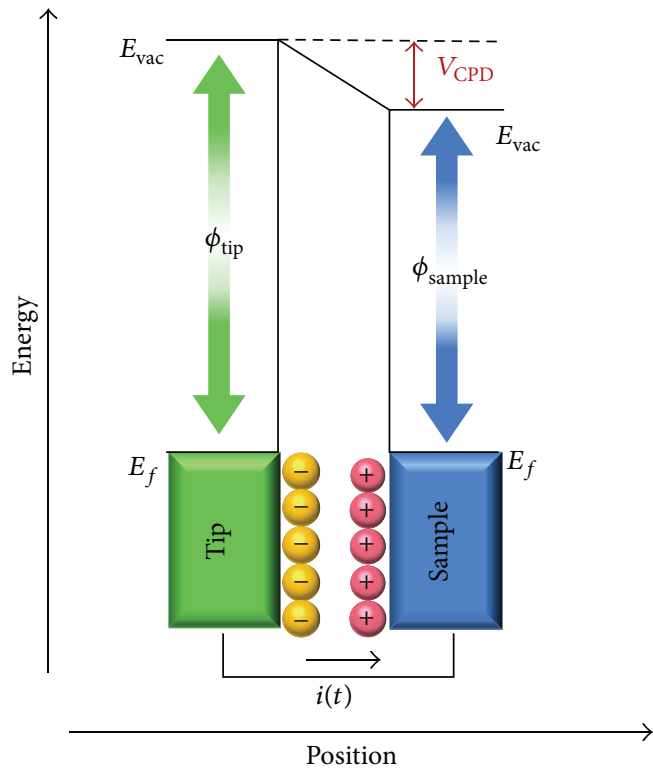

(c)

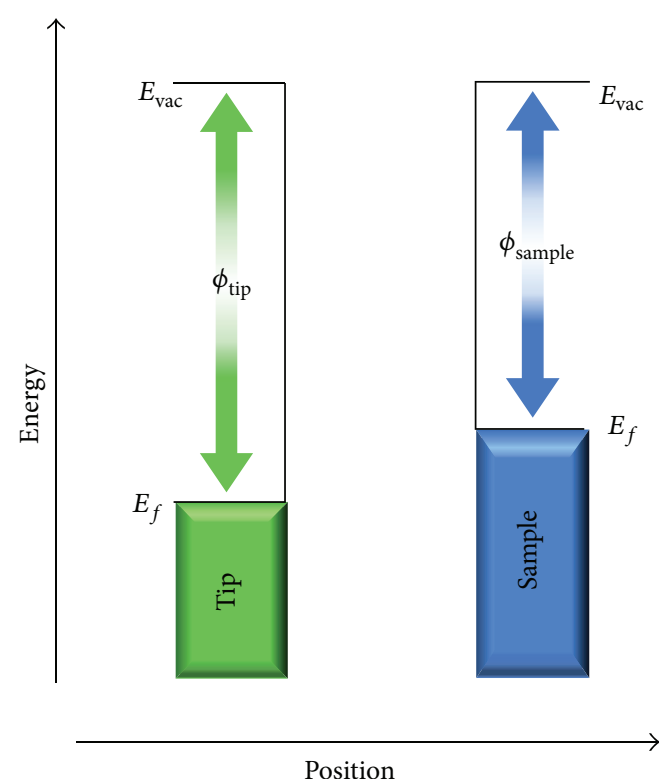

(b)

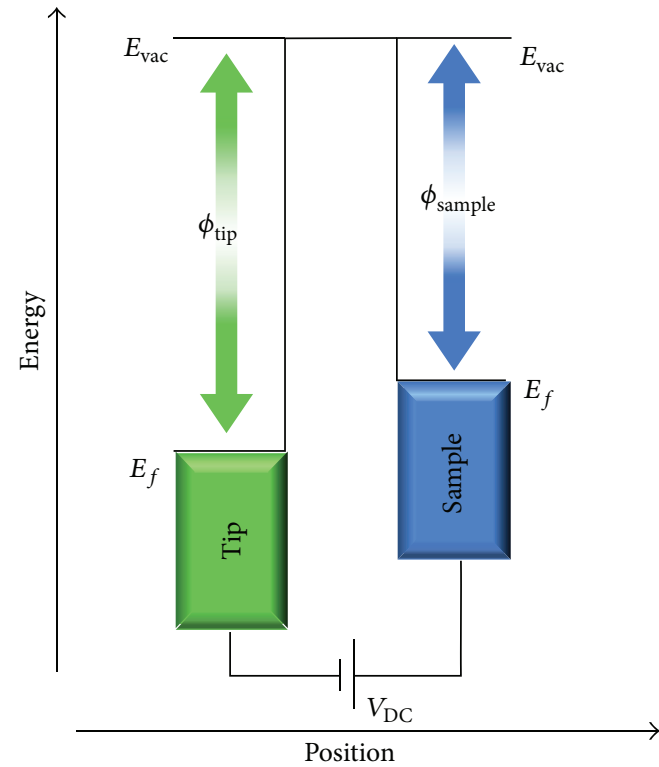

(d)

FIGURE 1: (a) Schematic diagram of general Kelvin method on electric circuit. (b-d) Electronic energy levels of the sample and AFM tip for three cases: (b) the tip and the sample are separated with no electrical contact, (c) the tip and the sample are in electrical contact, and (d) the external bias $\left(V_{\mathrm{DC}}\right)$ is applied between the tip and the sample to nullify the CPD and, therefore, the tip-sample electrical force. $E_{\mathrm{vac}}$ is the vacuum energy level and $E_{f}$ is the Fermi energy level. Figures reproduced with permission from [2], (c) 2011 Elsevier.

\section{Applications of KPFM}

3.1. Characterization of Electrical Properties in Various Nanomaterials. KPFM has been widely used to measure work functions of various materials at atomic or molecular scales. Unlike other detection methods, KPFM enables characterization of local electrical properties at subtle and complex areas such as interfaces in nanomaterials and junctions in semiconductor devices because of its high spatial resolution. The capacity for performing nanoscale electrical characterization is supported by the use of a conducting cantilever with a sharp tip (10 25 $\mathrm{nm}$ in radius). The following section reviews some applications of high-resolution KPFM to the characterization of the electrical properties of nanomaterials.

3.1.1. Sensing the Surface Potential of Nanoparticles. Nanoparticles mean very small particles having the size ranging from 1 to several hundred nanometers. Nanoparticles have been widely used in many areas such as biomedical [3941], optical [42-44], and electronic fields [41, 45]. KPFM is 


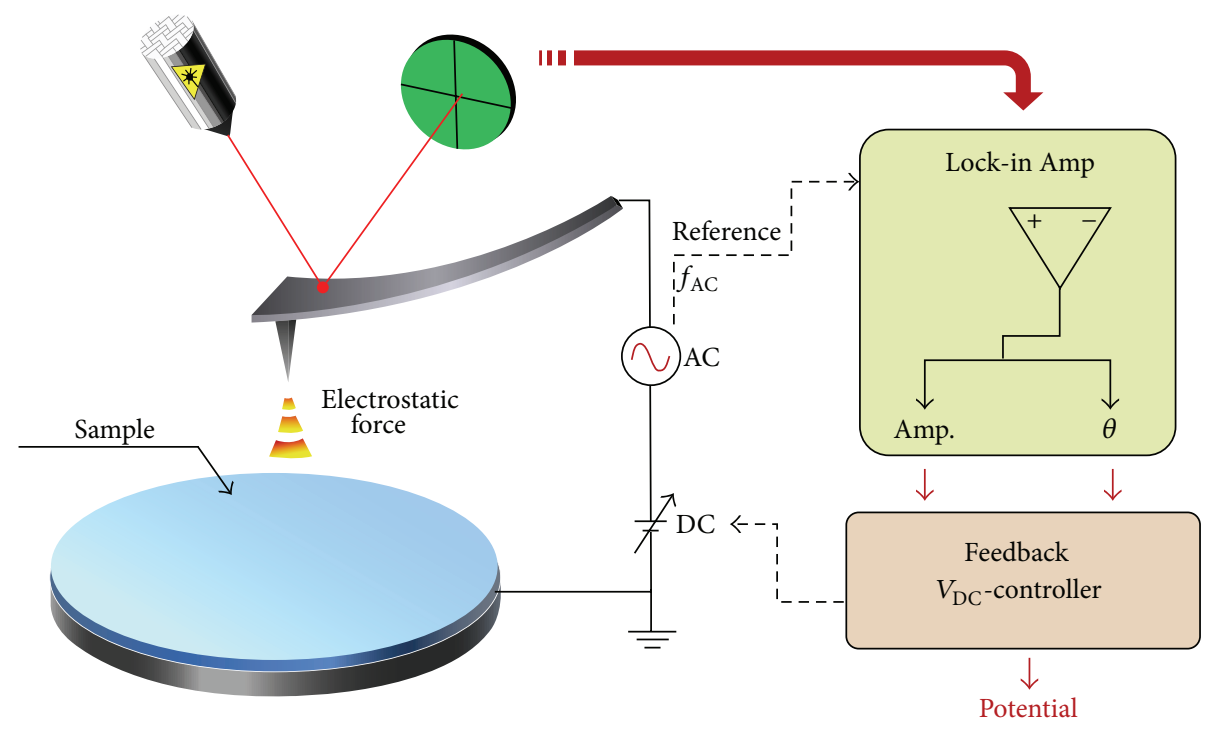

FIGURE 2: Block diagram of general KPFM system.

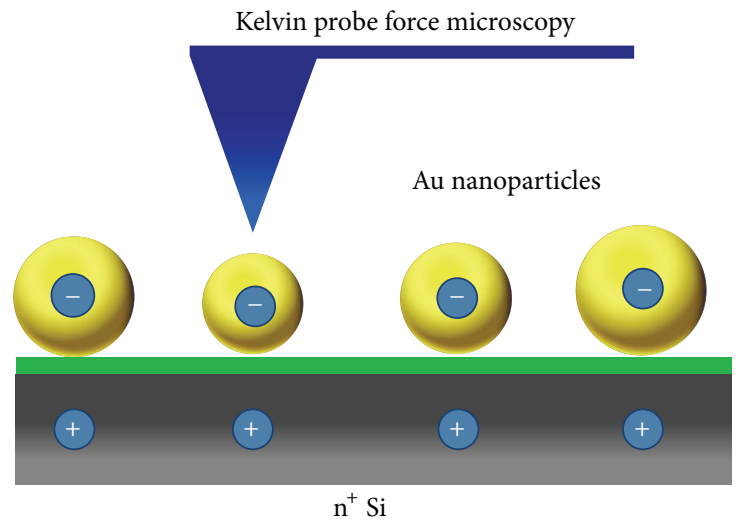

(a)

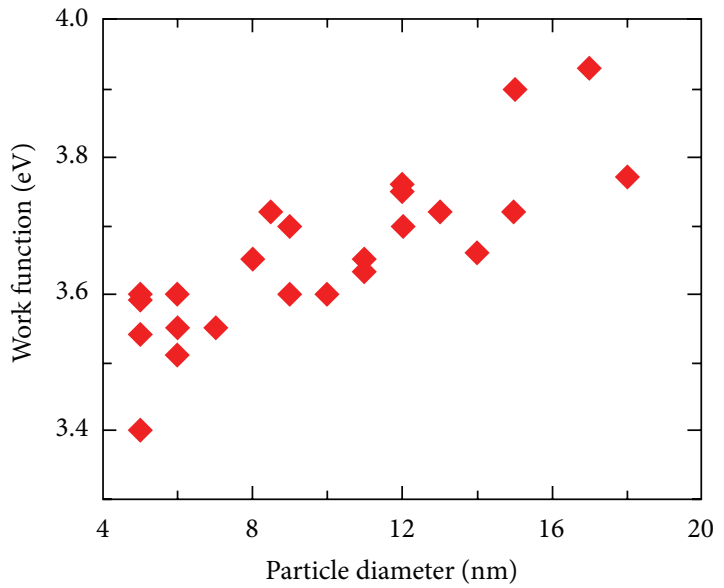

(b)

FIGURE 3: (a) Schematic diagram of the KPFM measurements of the AuNPs deposited on a silicon substrate covered with a grafted organic monolayer. (b) A plot of surface potentials as a function of AuNP diameter. Figures reproduced with permission from [28], (C) 2015 ACS.

one of the promising analytical tools to measure the surface properties of nanoparticles and monitor interaction between nanoparticles and other substances. For instance, accurate detection of the surface potential of nanoparticles can help one understand how the interactions between nanoparticles and neighboring molecules proceeds. In particular, gold nanoparticles (AuNPs) have been widely used in the field of chemistry and biomedical science [46] due to their unique optical [47, 48], electronic [49], and molecularrecognition properties [50]. Therefore, the surface potentials of AuNPs have been also studied extensively [51-53]. Zhang and coworkers investigated the variation in surface potential of the AuNPs with respect to particle size [28]. It was observed that when the AuNPs were reduced in size, their novel electrical properties became size-dependent. Figure 3 presents KPFM results for the surface potential variation of the AuNPs as a function of their size. The AuNPs were synthesized through reduction of gold salt by ascorbic acid and had a size distribution ranging from 3 to $\sim 25 \mathrm{~nm}$. A schematic setup regarding KPFM measurement is shown in Figure 3(a). The AuNPs spread on a silicon wafer covered by a monolayer of alkyl chains formed a tunnel junction. Figure $3(\mathrm{~b})$ reveals a consistent trend of the surface potential of the AuNPs with respect to their size. From observation, it was found that the surface potential increased as the size became bigger. For instance, the surface potential of a $10 \mathrm{~nm}$ AuNP charged with $\sim 5$ electrons was estimated to be only $\sim 3.6 \mathrm{eV}$. This report demonstrated that KPFM was able to accurately measure the work function of the AuNPs with different size. Because KPFM measurement is accurate enough to detect the small amount of electrons, the combination of KPFM and AuNPs is thought to be applicable 
to a nanoscale platform for chemical and biological sensing involving electron or ion exchange.

3.1.2. The Surface Potential Measurement of $2 D$ Layered Nanomaterials. To keep pace with proliferation of electronic devices field, research in regard to two-dimensional (2D) layered nanomaterials is currently at an advanced stage [54, 55]. Particularly, graphene is the most widely used 2D layered nanomaterial [56-58]. Because of its $\pi$-conjugation upon the sheet of $\mathrm{sp}^{2}$-hybridized carbon, the graphene has extraordinary electrical, mechanical, and optical properties. In particular, characterizing the electrical properties of graphene is necessary and of importance for understanding of the growth parameters of graphene and comprehension of the device functionality. To achieve this goal, accurate measurement of various electrical properties with high spatial resolution is required. Recently, Panchal and coworkers developed a graphene based FET that was a Hall bar structured device and analyzed its electrical properties using KPFM [23]. Figure 4 depicts KPFM results of the graphene Hall bar device, the electrical characterization of which were performed after surface potential calibration of a conductive Si cantilever tip with gold electrode. In this study, researchers analyzed and compared experimental results of lift-mode KPFM (Figure 4(a)), dual-frequency mode KPFM (Figure 4(b)), and electrostatic force spectroscopy (EFS). The surface potential measurement was conducted using each of three AFM techniques over the range of the applied biased voltage $\left(V_{\mathrm{ch}}\right)$. In the case of lift mode, the surface potential of graphene was measured as typically $27.6 \%$ lower than the actual $V_{\text {ch }}$. Otherwise, in the case of dual-frequency mode KPFM and EFS, the surface potential of graphene was measured as $4.4 \%$ and $7.8 \%$ higher than the actual $V_{\mathrm{ch}}$, respectively. From the results, it was found that dual-frequency mode KPFM and EFS were more accurate technique than lift mode. In addition, researchers explained that such inaccuracy of the lift-mode result was due to weak interaction between the cantilever tip and the sample separated with a lift height.

Despite many advantages of graphene, practical applications to nanoelectronic devices are currently at a slow pace, because graphene lacks a bandgap structure $[55,59]$. The bandgap of a material is a key property for enabling current regulation of nanoelectronic devices. On the other hand, molybdenum disulfide $\left(\mathrm{MoS}_{2}\right)$ is also a frequently studied 2D layered nanomaterial [60-62]. Moreover, some researchers predicted that $\mathrm{MoS}_{2}$ would be much better suited for designing electronic components [55]. Similar to graphite, bulk $\mathrm{MoS}_{2}$ also has layered structure, and its electrical properties change with respect to the thickness of $\mathrm{MoS}_{2}[63,64]$. However, the correlation between the electrical properties and the thickness of $\mathrm{MoS}_{2}$ has not been deeply studied yet. Ochedowski and coworkers fabricated single and multilayered $\mathrm{MoS}_{2}$ structure using chemical vapor deposition and successfully measured the surface potential of the $\mathrm{Au}-\mathrm{MoS}_{2}$ structure with various layers [29]. Figure 5 represents a KPFM study of a gold contact patterned on single-layered $\mathrm{MoS}_{2}$. A gold contact pattern was used to calibrate the work function of a conductive $\mathrm{Si}$ cantilever tip. Despite the calibration of KPFM tip, they determined the quantitative surface potentials for single-layered $\mathrm{MoS}_{2}$, bilayered $\mathrm{MoS}_{2}$, and $\mathrm{MoS}_{2}$ of a few layers. In Figure 5(d), the surface potential was measured to be $\sim 4.27 \mathrm{~V}$ for singlelayered $\mathrm{MoS}_{2}, \sim 4.37 \mathrm{~V}$ for a few layers of $\mathrm{MoS}_{2}$, and $\sim 4.89 \mathrm{~V}$ for gold. By using the known work function of gold $\left(\phi_{\mathrm{Au}}=\right.$ $5.10 \mathrm{eV}$ ), researchers formulated a statistical analysis of the work function according to the following equation: $\phi=$ $5.10 \mathrm{eV}-e \cdot\left(\mathrm{CPD}_{\mathrm{Au}}-\mathrm{CPD}_{n \mathrm{MoS}_{2}}\right)$. The work function of singlelayered $\mathrm{MoS}_{2}(\phi=4.49 \pm 0.03 \mathrm{eV})$ and a few-layered $\mathrm{MoS}_{2}$ $(\phi=4.59 \pm 0.03 \mathrm{eV})$ could be calculated.

As described above, the surface potentials of various nanomaterials can be measured using KPFM. Realization of such measurements at the nanoscale can trigger development of a sensing platform using a combination of KPFM and nanomaterials or electrical nanodevice. Actually, some experimental results regarding KPFM-based sensors have started to be reported recently. Details regarding this will be discussed later.

\subsubsection{Surface Potential Mapping of Oligonucleotides. For} understanding various biomolecular interactions, it is of great significance that one can measure the morphological structure and the surface potential of biomolecules, including proteins, polynucleotides, metabolites, and other cellular components [16, 65-67]. In general, various functions of these biomolecules are mediated by electrostatic interactions with other molecules in many biological systems. Up to now, many researchers have reported the surface potential and the electrostatic interactions between biomolecules by using an analytical method (i.e., zeta potential) [68-70]. However, this method has a limitation that it cannot be used to measure the specific surface potential of biomolecules coexisting with other molecules in solution. According to the literature, KPFM is a promising technique to directly detect the electrostatic interactions with single-molecule resolution and it enables measurement of the surface potential of biomolecules for biomedical applications [71, 72].

Electrostatic force can mediate the interactions between oligonucleotide and other biomolecules [73]. The electrostatic force between some biomolecules is closely related to their surface potentials. The surface potentials of oligonucleotides (short DNA or RNA molecules) play a key role in determining their molecular structure and functions such as hydrophobicity and hybridizations [74]. However, accurate measurement of the surface potential of individual biomolecules is still lacking and challenging. Recently, Leung and coworkers succeeded in the mapping of the surface potential of biological molecules (avidin and DNA) on a silicon substrate, with high sensitivity [17]. The avidin molecules were $\sim 10 \mathrm{mV}$ in surface potential, indicating that they were more positive compared to the substrate in Figure 6(a), whereas the DNA strands were $\sim-150 \mathrm{mV}$, as shown in Figure 6(b). From this study, it was proved that KPFM technique can measure the surface potential of biomolecules at single-molecule level. These results imply that KPFM measurement is able to help researchers understand the mechanism of how physicochemical factors influence biomolecular interactions. In this work, however, the silicon substrate was thought to be not suitable for measuring the surface 

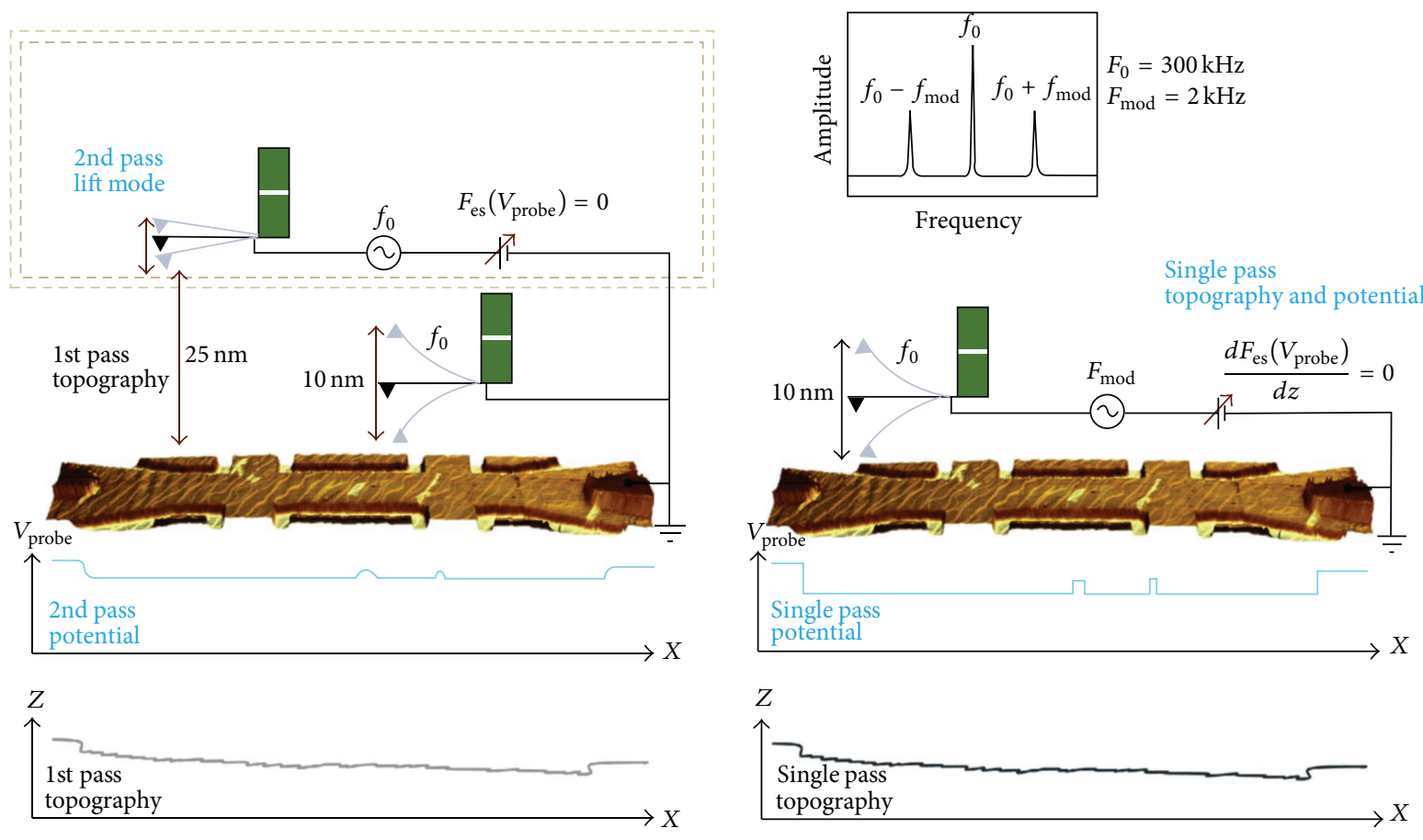

(a)

(b)

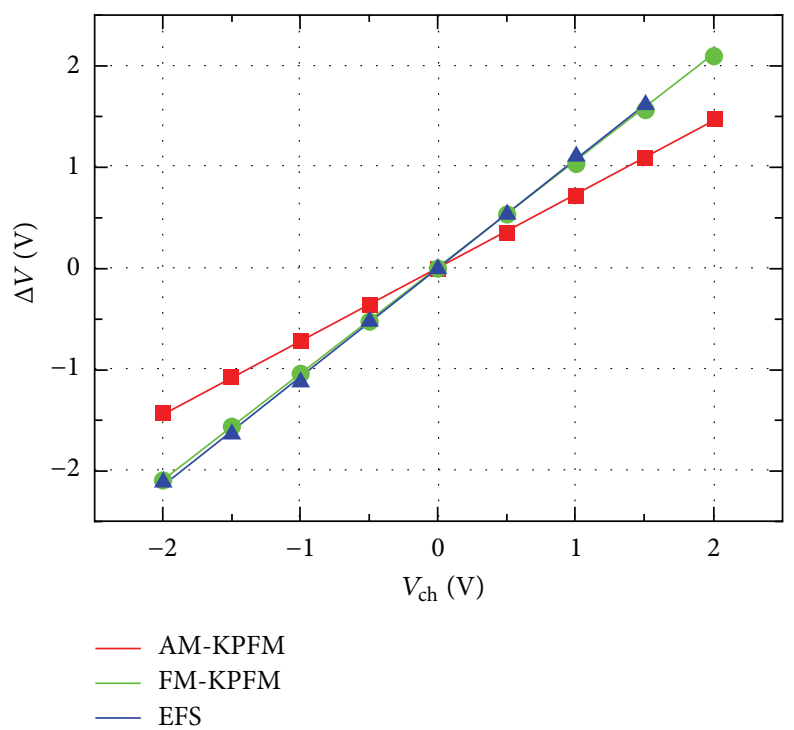

(c)

FIGURE 4: (a-b) Schematic diagrams of the experimental techniques. (a) Lift-mode KPFM and (b) dual-frequency mode KPFM; the topography of the graphene Hall bar is superimposed with surface potential maps on a 3D image. The plots show characteristic profiles (i.e., surface potential on top and topography on bottom along the horizontal line in the center of the image). (c) Normalized SP values as measured by AM-KPFM, FM-KPFM, and EFS techniques on the left gold electrode in dependence on the voltage applied to the same gold electrode. Figures reproduced with permission from [23], (c) 2013 NPG.

potentials of DNA owing to the weak affinity between DNA and substrate. Instead, mica substrate was preferentially suggested as the relevant substrate, because various substances could be easily attached to it, maintaining their native structures. In the next work, Leung and coworkers measured the topology and the surface potential of DNA strands after they were spread on a mica substrate [18]. In addition, both lift-off mode and dual-frequency mode were used to measure the surface potential in this work, the result of which is shown in Figures 6(c) and 6(d). In a viewpoint of spatial resolution, it was proved that the dual-frequency mode is more advantageous for acquiring the surface potential image with high spatial resolution.

3.1.4. Mapping the Surface Potential of Amyloid Fibrils. Amyloid fibrils, which transform from their native protein 


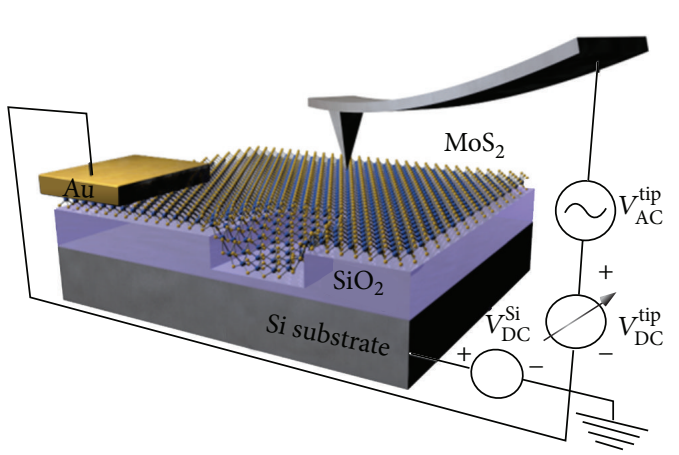

(a)

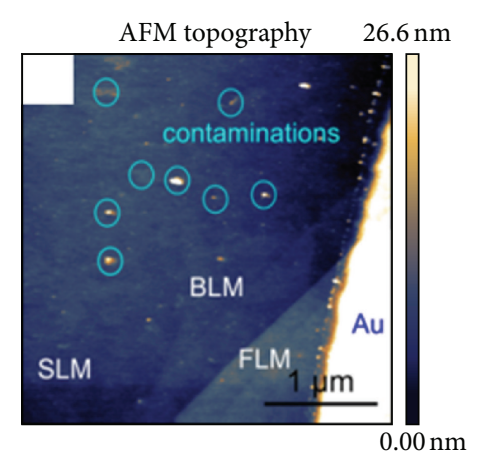

(b)

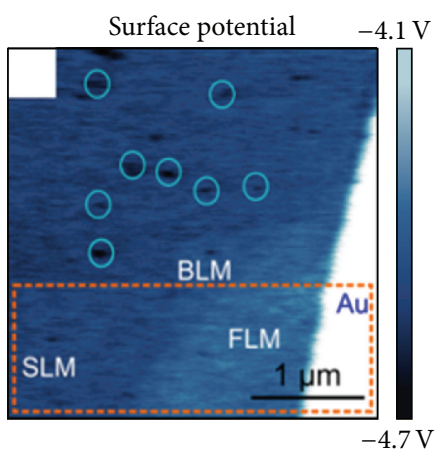

(c)

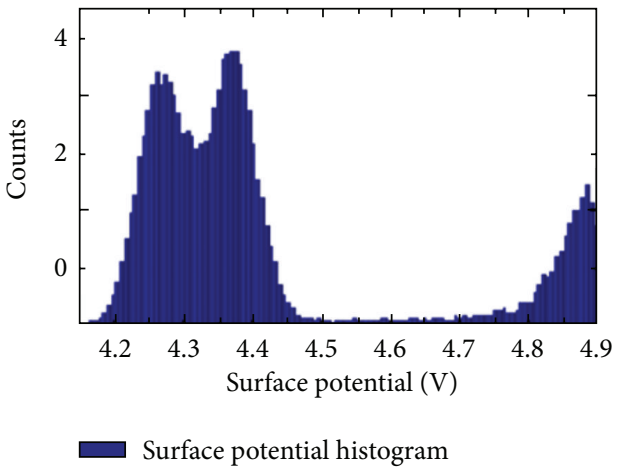

(d)

FIGURE 5: (a) Schematic representation of the KPFM setup and the $\mathrm{MoS}_{2}$ sample with the $\mathrm{RIE} \mathrm{SiO}_{2}$. (b) $\mathrm{AFM}$ image of $\mathrm{MoS}_{2}$ flake on $\mathrm{SiO}{ }_{2}$ with a gold contact (height $=20 \mathrm{~nm}$ ). The topography shows areas with contaminations due to processing. (c) The corresponding surface potential image to (b). The surface potential of $\mathrm{MoS}_{2}$ is increasing with increasing layer thickness; contaminations can be clearly distinguished in the surface potential image. (d) Surface potential histogram of the box marked in (c). Figures reproduced with permission from [29], (c) 2014 Belistein.

structure to $\beta$-sheet rich structures, are related to multiple degenerative disorders such as Alzheimer's, Parkinson's, and Creutzfeldt-Jakob diseases [75-77]. Amyloid peptides, proteins, and fibrils may be coupled with other macromolecules such as DNA, lipid, and polysaccharides by electrostatic force $[78,79]$. These aggregates contribute to onsets of various diseases. Accordingly, it is necessary to measure electrostatic properties of amyloid fibrils and electrostatic interactions with other biomolecules for understanding some mechanisms regarding cytotoxicity [80]. The following paragraphs review some examples in regard to characterization of the electrical properties of amyloid fibrils and the molecular interactions with other biomolecules.

To scrutinize how lipid membrane composition play a role in fibril formation and cytotoxicity, Drolle and coworkers investigated the effect of cholesterol on the interaction of model lipid membranes with amyloid beta $(\mathrm{A} \beta)$ peptide [30]. The surface potentials of lipid membranes with/without cholesterol were measured. Figures $7(\mathrm{a})$ and $7(\mathrm{~b})$ depict surface potential images of both the pure lipid monolayer and the lipid monolayer with $20 \%$ cholesterol. It was shown that the pure lipid monolayer was smooth and had a uniform and featureless surface potential, whereas the lipid monolayer with $20 \%$ cholesterol had a surface potential difference of $61 \pm$ $8 \mathrm{mV}$. This result indicates that the cholesterols might serve as anchor molecules for binding amyloidogenic molecules to membranes. These $\mathrm{A} \beta$ peptides-lipid complexes are thought to be a cause providing the cytotoxicity of amyloid fibrils.

Amyloid fibrils retain a capacity for being combined with nanomaterials as well as biomolecules to form some complexes or aggregates. For instance, $\mathrm{Ma}$ and coworkers reported the synthesis of composites being composed of amyloid fibrils and AuNPs and the KPFM analysis of them [31]. To investigate the effect of nanomaterials on fibrillation, A $\beta$ 25-35 peptides were incubated with gold nanoparticles (AuNPs) at room temperature for $5 \sim 12$ days. The KPFM analysis was performed in order to investigate how the electrical properties of the A $\beta 25-35$ fibrils with and without AuNPs change. As shown in Figures 7(c) and 7(d), it was found that, without AuNPs, the A $\beta 25-35$ peptides formed into long fibrils depending on incubation time; with AuNPs, short fibrils and bundles coexisted, indicating that there was bundle conjugation. KPFM was used to measure electrical phase shifts of the pure A $\beta 25-35$ fibrils and the AuNPs/A $\beta$ 2535 complexed fibrils, the result of which is shown in Figures $7(\mathrm{c})$ and $7(\mathrm{~d})$. The topography and phase shift images of the pure $A \beta 25-35$ fibrils were collected at a sample bias of $-3 \mathrm{~V}$ and a lift height of $60 \mathrm{~nm}$. The fibrils without AuNPs had a similar phase shift of $\sim 0.1^{\circ}$ relative to the Si substrate. However, the A $\beta$ 25-35 fibrils with AuNPs had a height of $20 \sim 30 \mathrm{~nm}$ and a phase shift of $\sim 1.0^{\circ}$ relative to the Si substrate at the bias of $-2 \mathrm{~V}$ and a lift height of $40 \mathrm{~nm}$. From these 

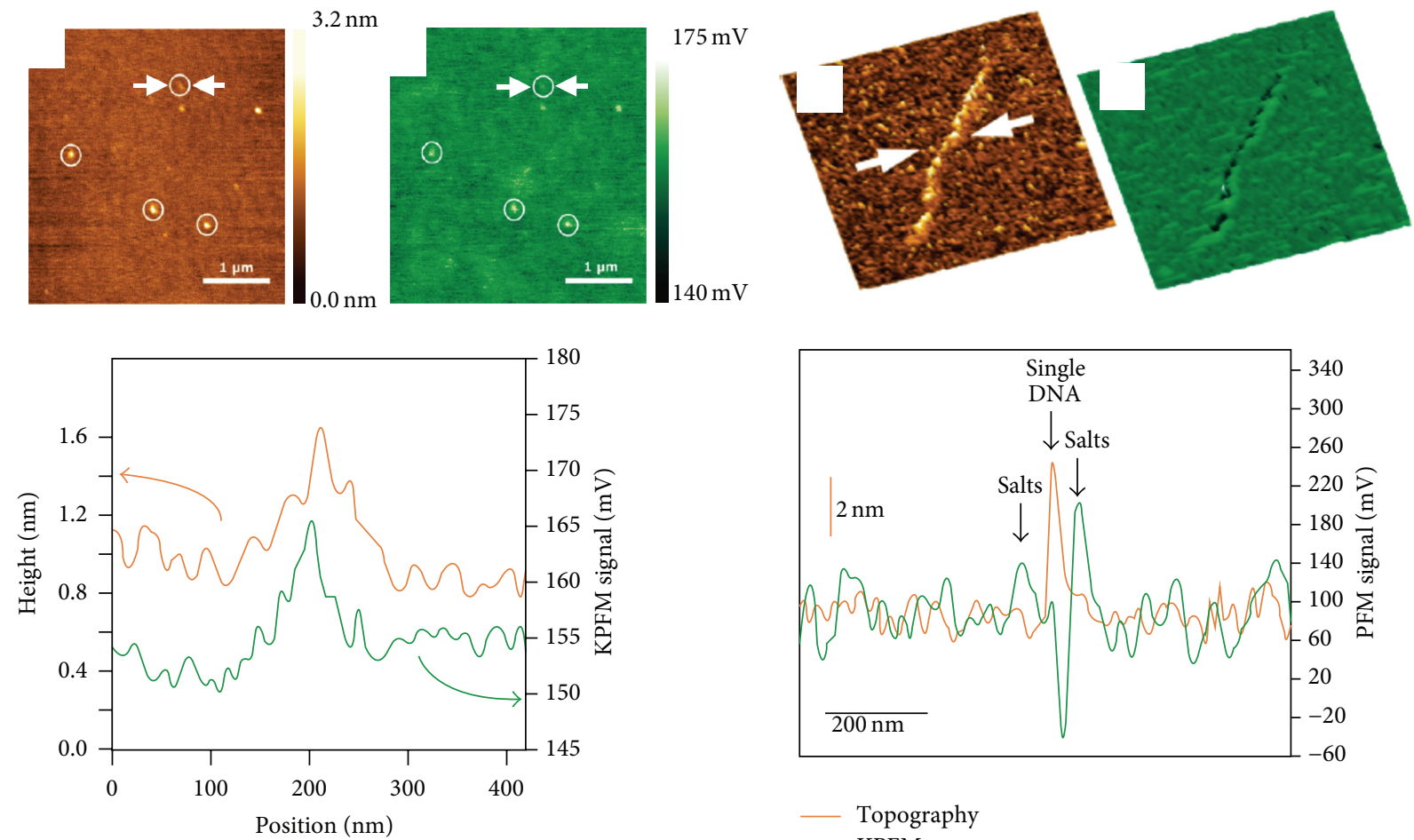

Topography

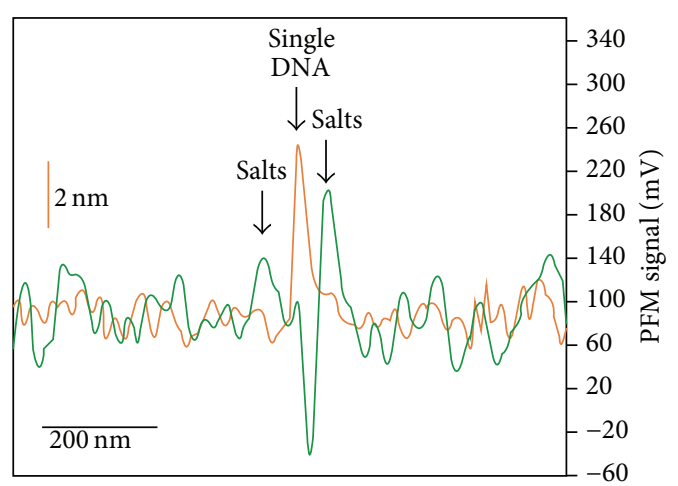

- KPFM

(a)

(b)
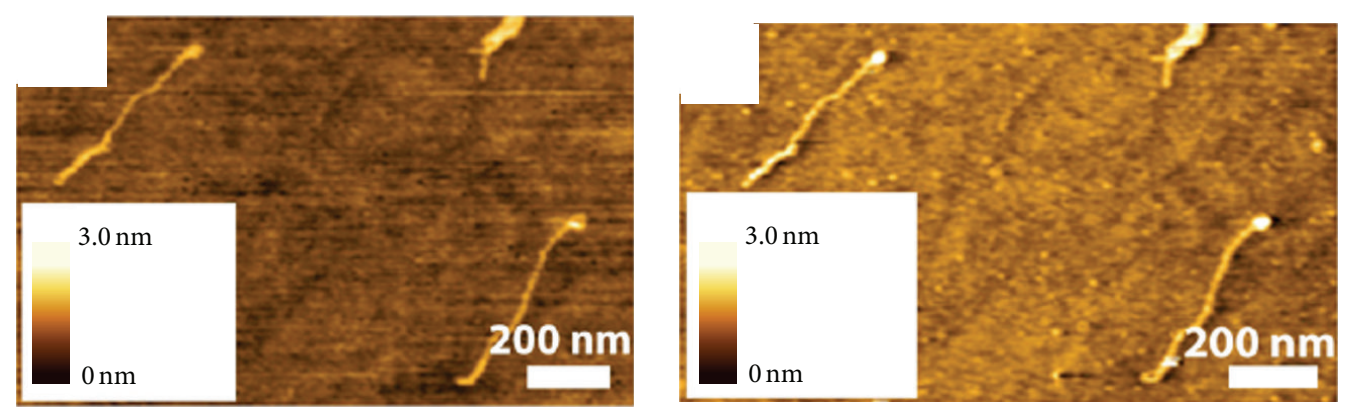

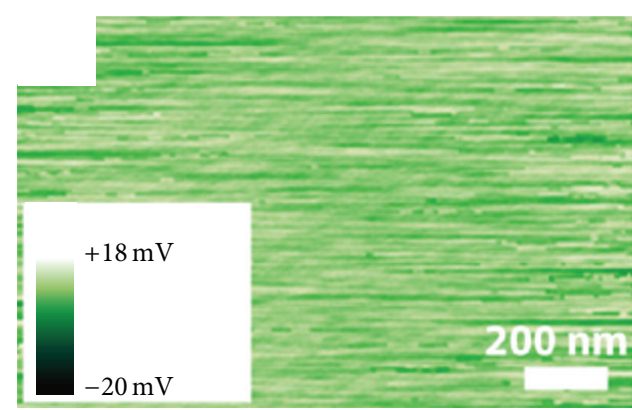

(c)

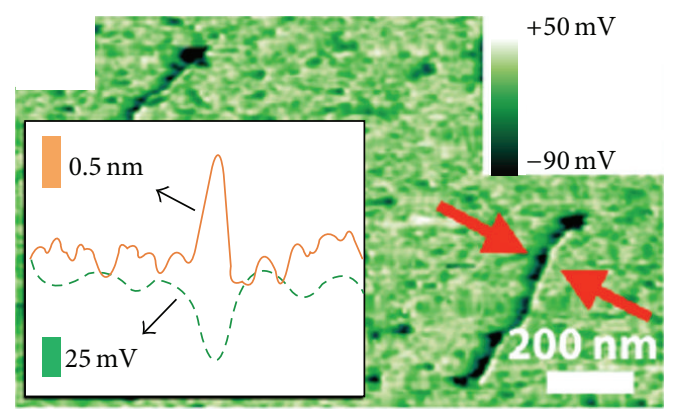

(d)

FIGURE 6: The AFM topographic image and the corresponding KPFM image of (a) avidin and (b) DNA molecules. Line profiles corresponding to the topographic and KPFM data at the location indicated by the arrows. Figures reproduced with permission from [17], (C) 2009 ACS. (c) Topography and corresponding KPFM image acquired in lift mode and (d) dual-frequency mode for single DNA molecules. Figures reproduced with permission from [18], (c) 2010 AIP. 

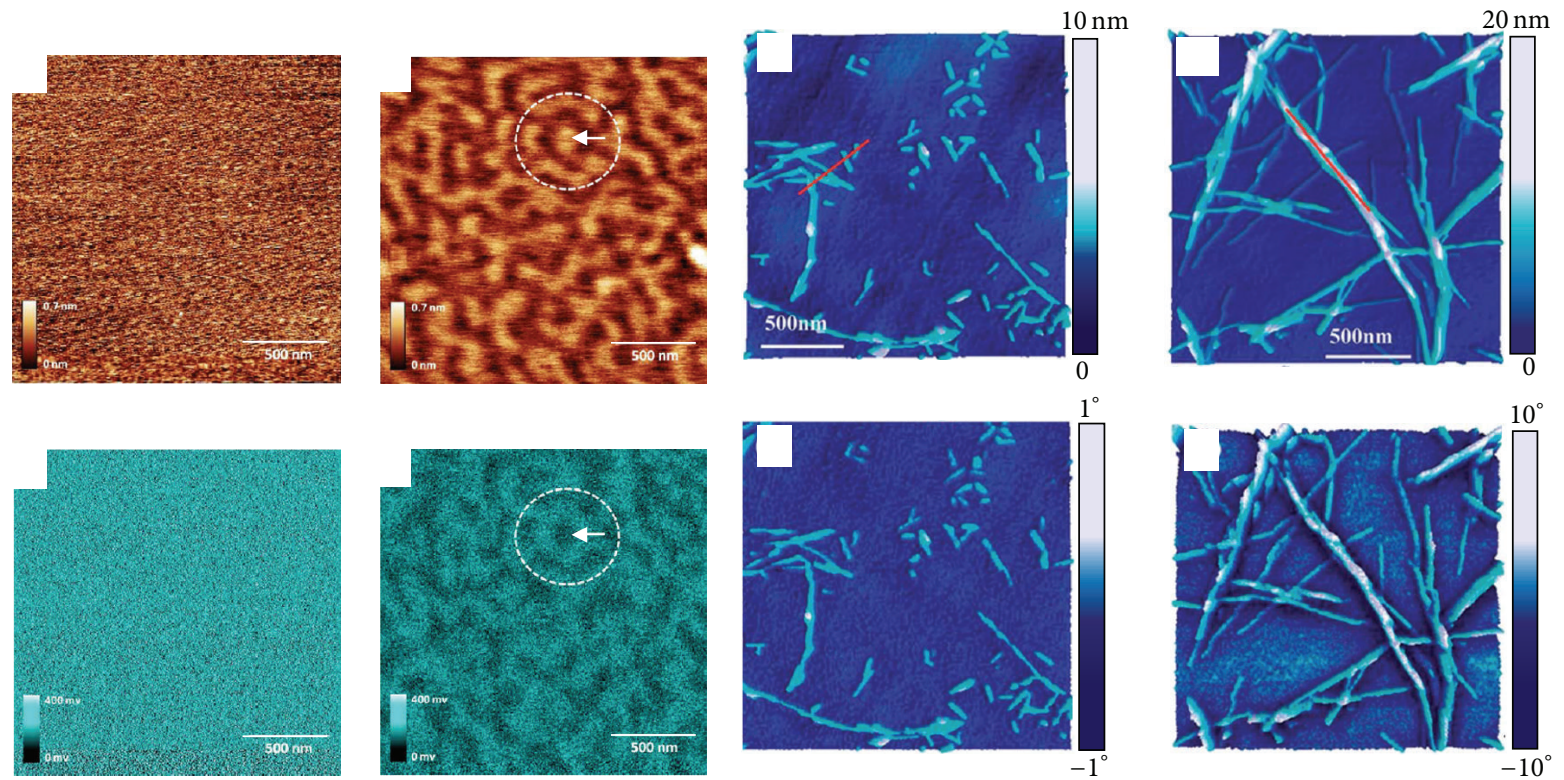

(a)

(c)

(d)

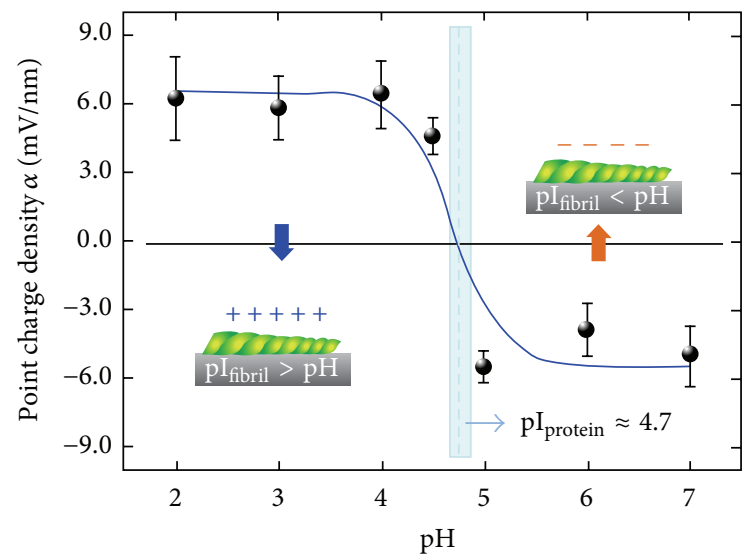

$\mathrm{pH} 3$

pH 6

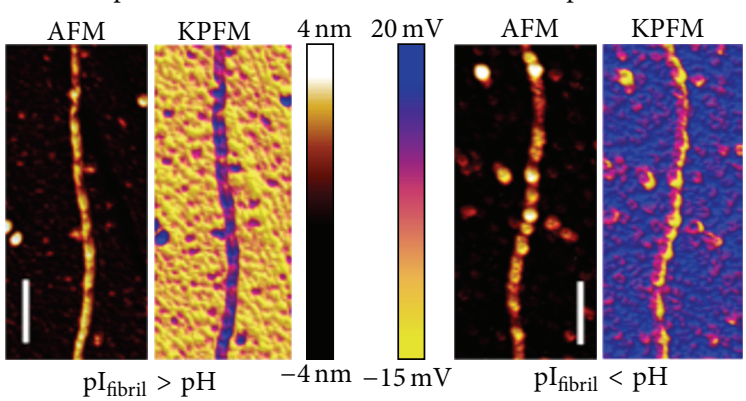

(e)

FIgURE 7: (a) The AFM and corresponding KPFM images of lipid monolayers without cholesterol. (b) The AFM and corresponding KPFM images of lipid monolayers with $20 \%$ cholesterol. Figures reproduced with permission from [30], (c) 2012. The Biophysical Society. (c) The topography and EFM images of $\mathrm{A} \beta_{25-35}$ deposited on Si substrate incubated 12 days after preparation. (d) The topography and EFM images of $\mathrm{A} \beta_{25-35}+$ AuNPs deposited on Si substrate incubated 10 days after preparation. Figures reprinted with permission from [31], () 2013 RSC. (e) The surface charge distribution of amyloid fibril as a function of the $\mathrm{pH}$ of buffer solution in which amyloid fibril is prepared. Figures reproduced with permission from [19], (c) 2012 AIP. 
results, it is plausible that KPFM is useful for monitoring the conformational change and the electrical properties of amyloid fibrils during self-assembly with nanomaterials.

$\beta$-lactoglobulin $(\beta-\lg )$ has attracted much attention as an alternative amyloid precursor used to investigate the fibrillation mechanism and to fabricate various composites because of its inexpensive price and easy fabrication [8184]. The $\beta$-lg, a protein derived from bovine milk, is able to form fibrillary structure at low $\mathrm{pH}(\mathrm{pH} 2)$ [85]. Lee and coworkers studied the electrical properties of the $\beta$ lg fibrils using KPFM, which enabled the visualization and mapping of surface potential distribution of a single amyloid fibril (Figure 7(e)) [19]. The result revealed that the surface potential of an amyloid fibril was critically dependent on not only its structural conformation but also solvent $\mathrm{pH}$. The surface potential of amyloid fibrils ranged from $12 \mathrm{mV}(\mathrm{pH} \sim$ 2) to $-12 \mathrm{mV}$ ( $\mathrm{pH} 7$ ), depending on the $\mathrm{pH}$ of buffer solution. Moreover, in the case of $\mathrm{pH}<\mathrm{pI}(\sim 5.0)$, the $\beta$-lg had a conformation like protofilaments. On the contrary, the $\beta$-lg had a complex structure of $\beta$-lg oligomer-binding protofilaments that had irregular topological height, in the case of $\mathrm{pH}>\mathrm{pI}$. It was remarkably observed that the $\mathrm{pH}$-dependent electrostatic property of amyloid fibrils was responsible for binding affinity between the amyloid oligomers and the amyloid fibrils.

Whether the regrowth of decomposed amyloid fibrils is possible and how likely this is has attracted increasing interest during the past few decades due to clinical importance. Lee and coworkers investigated the decomposition characteristics and the regrowth behavior of the amyloid fibrils using ultrasound and KPFM [20]. They observed that the contour length of decomposed amyloid fibrils was inversely proportional to the duration time of ultrasound, and the decomposed fibrils could be regrown by addition of monomeric protein. From the KPFM analysis, it was known that the regrown fibrils exhibited electrical properties similar to those of the original fibrils. As described above, KPFM indeed was a good analytical tool for monitoring the conformational change and the electrical properties of disease-related biomolecules after clinical treatment like ultrasound.

\subsubsection{Single-Molecule Recognition of Biomolecular Interac-} tions. Single-molecule level analysis of biomolecular interactions such as hybridization of DNA strands, ligandreceptor binding, antigen-antibody binding, and enzymatic reaction is important in biological technology [86-89]. These biomolecular interactions have significant effects on biological phenomena such as genomic processes, signal processes, immune system function, and other biological functions. Although conformational structures of various biomolecules have been reported, electrostatic interactions between biomolecules have not yet been fully elucidated. Furthermore, how the surface potential of individual molecules is substantially related to their electrostatic force and binding affinity is still unclear. KPFM has emerged as a remarkable tool for monitoring a variety of interactions between ligands and receptors, of biomolecules on nanomaterial surfaces by measuring their surface potential changes $[87,90,91]$. The following paragraphs review some examples in regard to investigations of single-molecular recognition and bimolecular interactions via KPFM techniques.

Single-molecule level detection of ATP-driven activation of tyrosine kinase and inhibition by Gleevec is a very successful example of monitoring disease-related biomolecular interactions using KPFM [26]. Protein kinases play a significant role in regulating cellular functions, including signal transduction. Moreover, mutations of tyrosine kinases are the cause of initiation or progression of several cancers. It is difficult to detect and sense such interactions between a protein kinase and a small ligand (i.e., adenosine- 5 ' -triphosphate (ATP) and inhibitor) using conventional methods owing to limited spatial resolution. However, KPFM is able to clearly distinguish whether a single ATP is bound on a single protein kinase or not. Park and coworkers investigated the singlemolecule level detection of the ATP-driven activation and the inhibition of tyrosine kinase using KPFM [26]. When the protein kinases were exposed to only the ATP, and the mixture of ATP and inhibitor (Gleevec), the surface potential profiles revealed interesting results. When exposed to only the ATP, the protein kinases exhibited a surface potential negatively increased by $\sim-25 \mathrm{mV}$ in comparison with the innate protein kinase-presumably due to strong negative charges of ATP. However, when exposed to the mixture of ATP and inhibitor, they had a similar surface potential to that of the innate protein kinase due to neutral charge of inhibitor. That indicates that the protein tyrosine kinase can be easily inhibited by addition of Gleevec. These results suggest that KPFM technique allows for identification of biomolecular interaction between a small ligand and a single biomacromolecule and provides information of its binding affinity at single-molecule resolution. Accordingly, KPFM may be regarded as a single-molecule drug-screening toolkit capable of providing insights into the efficacy of designed drugs, including nanomedicines.

KPFM also allows one to measure the surface potential of plasma membranes [92]. The cell plasma membrane, which separates the interior of cells from the extracellular environment, is involved in various cellular functions such as cell adhesion and signaling. Depending on physiological environments, the surface potential of the plasma membrane is expected to be changed. Pan and coworkers tried to perform the topological imaging and the surface potential measurement of plasma membranes using KPFM. To measure the surface potential of plasma membrane, the PC12 cells, a cell line derived from a pheochromocytoma of the rat adrenal medulla, were used. The measured surface potential of the PC12 cells was $\sim 5 \pm 0.15 \mathrm{~V}$, indicating that there were some variations in surface potential with respect to the measuring location in plasma membrane. These variations are thought to be due to diversity of membrane components such as lipids, phospholipids, sterols, carbohydrates, and proteins. They calculated the skewness values from the surface potential images and then identified three types of distribution patterns in terms of the skewness. In addition, they studied how stimulation by various molecules $\left(\mathrm{H}_{2} \mathrm{O}_{2}\right.$, dopamine, or $\left.\mathrm{Zn}^{2+}\right)$ affects the surface potentials of cell membranes. The skewness value of the control PC12 cells was $-0.21 \pm 0.21$. When a 
stimulation applied, the skewness was estimated to be $0.25 \pm$ $0.15,0.37 \pm 0.14$, and $0.32 \pm 0.1$ for the cells treated with $\mathrm{H}_{2} \mathrm{O}_{2}$, dopamine, and $\mathrm{Zn}^{2+}$, respectively. As described above, it was proved that KPFM was useful for examining some variations in surface properties of the cell membranes by addition of some drugs.

\subsection{Direct Detection of Biological/Nanomolecules for Biosensor Applications}

3.2.1. Electrical Properties Characterization of Field-Effect Transistors. KPFM method can be also applied to the optimization and characterization of the surface potential of material surfaces for developing electronic devices, including semiconductors and field-effect transistors (FETs) [93, 94]. In the development of bioelectronic devices, measuring the degree of surface functionalization of biomolecules on devices or FETs is important for confirmation of their sensing ability. To our knowledge, many methods such as electron and fluorescence spectroscopy have been reported for estimating the surface properties of various devices. However, these methods have some limitations, such as difficulty in measuring the local electrical properties of surfaces and labeling with chemical reagents. KPFM is thought to be an excellent analytical tool to overcome these hurdles [16]. Tsai and coworkers investigated the surface properties of the asfabricated silicon nanowire-FET (SiNW-FET) using KPFM [32]. The surface topology of SiNW-FET device was obtained by SPM. Meanwhile, the corresponding surface potential images were collected at the source-drain voltage $=0 \mathrm{~V}$, the gain voltage $=0 \mathrm{~V}$, and the lift height $=30,60$, and $120 \mathrm{~nm}$, respectively (Figure 8(a)). When the lift height increased, the surface potential decreased and the sensitivity of FET device also got lower. In addition, to measure how efficiently the biomolecules were immobilized on the FET device, the average surface potentials were measured after immobilization of various biomolecules. The surface potential was estimated to be $4.832 \pm 0.004 \mathrm{~V}, 4.816 \pm 0.007 \mathrm{~V}$, and $4.870 \pm 0.013 \mathrm{~V}$ for the 3-(mercaptopropyl)trimethoxysilane (MPTMS)/SiNW-FET, the biotin/SiNW-FET, and the avidin/SiNW-FET, respectively.

Exfoliated graphene and graphite oxide, which have outstanding electrical, mechanical, and optical properties, were commonly used to develop electronic devices for various sensor applications $[95,96]$. Recently, it had been reported by Yan and coworkers that KPFM could be used to characterize local voltage drop in single functionalized graphene sheets (FGSs) under external bias condition [33]. They measured the surface potential of individual FGSs to determine their innate conductivity and the charge transport between FGSs and metal contacts. Figure 8(b) shows two-dimensional voltage drop images of the FGS, measured at the external bias of $2 \mathrm{~V}$ and the current of $0.68 \mathrm{~mA}$. Here, there was no significant potential drop between FGS and gold contacts. The voltage drop curve exhibited two particular slopes between the gold contacts regions, which may be related to their current density. In addition, it was observed that wrinkles of FGSs had a negligible effect on the voltage drops. These results indicate that KPFM method can give insight into investigating local electrical properties of functionalized graphene or graphene oxide sheets.

3.2.2. Label-Free Nucleotides Analysis. To scrutinize morphological structures of biomolecules and mechanisms underpinning interactions between them, it is important that the analytical tool has a capacity for visualizing various biomolecules at single-molecule resolution [97]. Some visualization methods for biomolecules have a critical disadvantage that these require labeling process by fluorescent or chemical reagents. The added molecules for labeling can have a positive or negative influence on the interaction between biological molecules, altering their innate characteristics. However, KPFM can acquire the visualization of biomolecules at singlemolecule level including structure and surface potential information, without any side effects caused by labeling or staining with fluorescent molecules [34].

Recently, Sinensky and Belcher developed label-free DNA nanoarray that was fabricated by dip-pen nanolithography (DPN) and analyzed by KPFM. To characterize hybridization behaviors in the DNA nanoarray, thiol-modified single stranded DNA probes were patterned into various shapes (lines and dots) on gold substrates by using DPN [34]. Figures 9(a)-9(c) depict KPFM results in regard to the DNA hybridization on the DNA nanoarray. When the probe DNA was exposed to complementary DNA, it was observed that the surface potential of individual dot was doubled $\left(\phi_{\text {probe }}\right.$ $=\sim 56 \mathrm{mV}, \phi_{\text {hybridized }}=\sim 110 \mathrm{mV}$ ). In addition, it was also found that the surface potential of individual dot was strongly affected by the scan height and the scan rate (Figure 9(d)). That indicates that seeking the optimal measuring condition prior to analysis is necessary to obtain the reliable surface potential in the DNA nanoarray.

To fabricate efficient and high-performance biosensor devices, it is essential to monitor the surface property change during the entire functionalization process of biomolecules [98]. For the last decade, various organic monolayers have attracted much attention because they could be used as linkers or anchors for immobilization of biological molecules in biosensor applications [99]. KPFM has been proposed as a way to quantitatively measure how much amount of linkers or biomolecules are immobilized on the nanomaterial or the device [100]. Richards and coworkers performed the immobilization of DNA on GaP substrate and measured the surface potential of the substrate surface before and after the immobilization [35]. The GaP surfaces patterned with 10, 20, and 30\% undecenoic acid (UDA) by PDMS stamp were prepared. Before the DNA immobilization, the sample treated with $10 \%$ UDA was $\sim 4 \mathrm{mV}$ in surface potential. It was found that as the UDA concentration increased, the surface potential also increased $(\phi=\sim 9 \mathrm{mV}$ at $30 \%$ UDA). They observed that, after the DNA immobilization, the surface potential of samples strongly depended on the UDA concentration, exhibiting $\phi=\sim 10.5 \mathrm{mV}, \sim-3.8 \mathrm{mV}$, and $\sim-4.0 \mathrm{mV}$ at $10 \%, 20 \%$, and $30 \% \mathrm{UDA}$, respectively (Figures 9(e) and 9(f)). This implies that the patterned UDAs on the $\mathrm{GaP}$ have different arrangement or conformation with respect to their concentration, resulting in some variation in conformation with the immobilized DNA. As described 

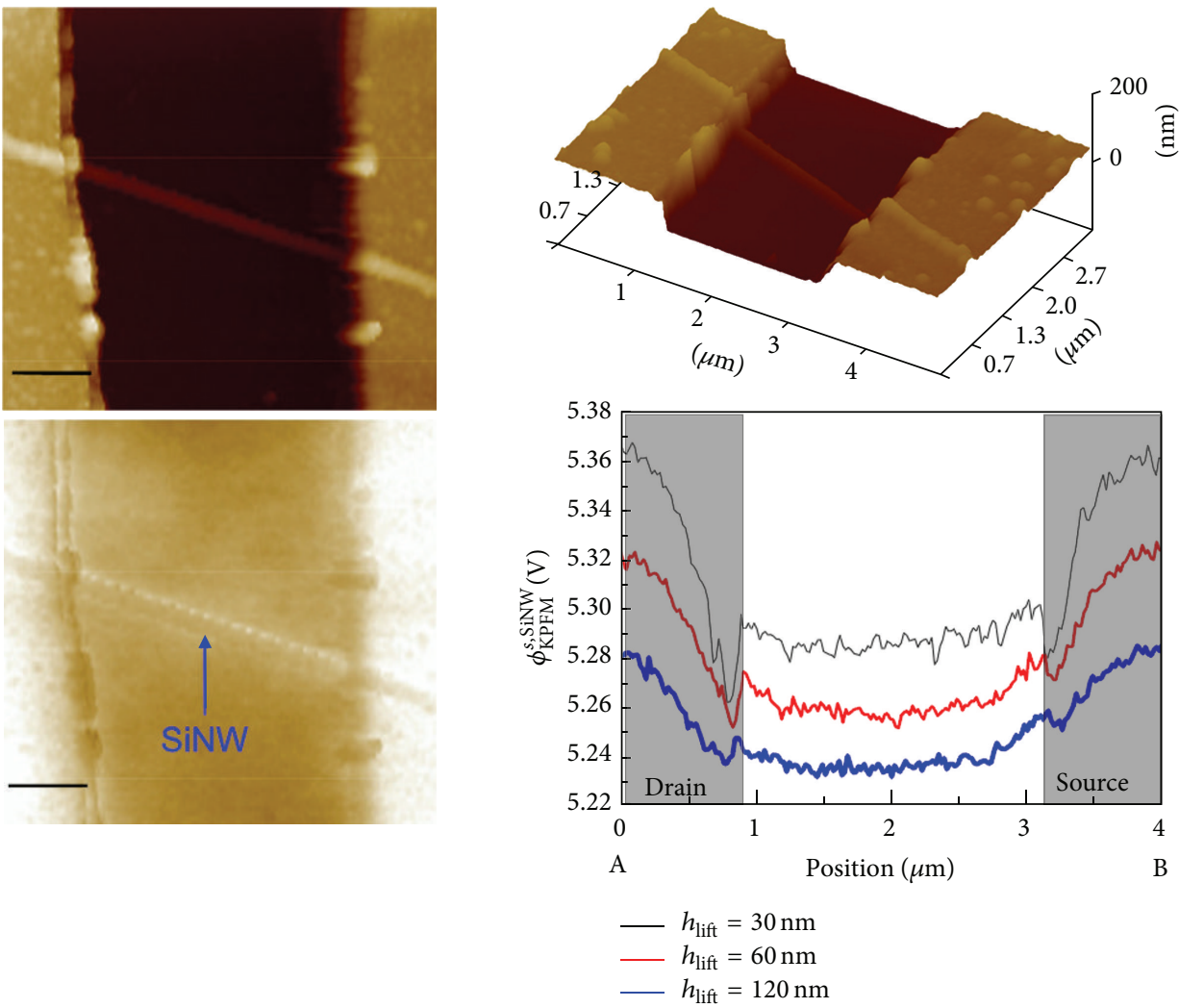

(a)

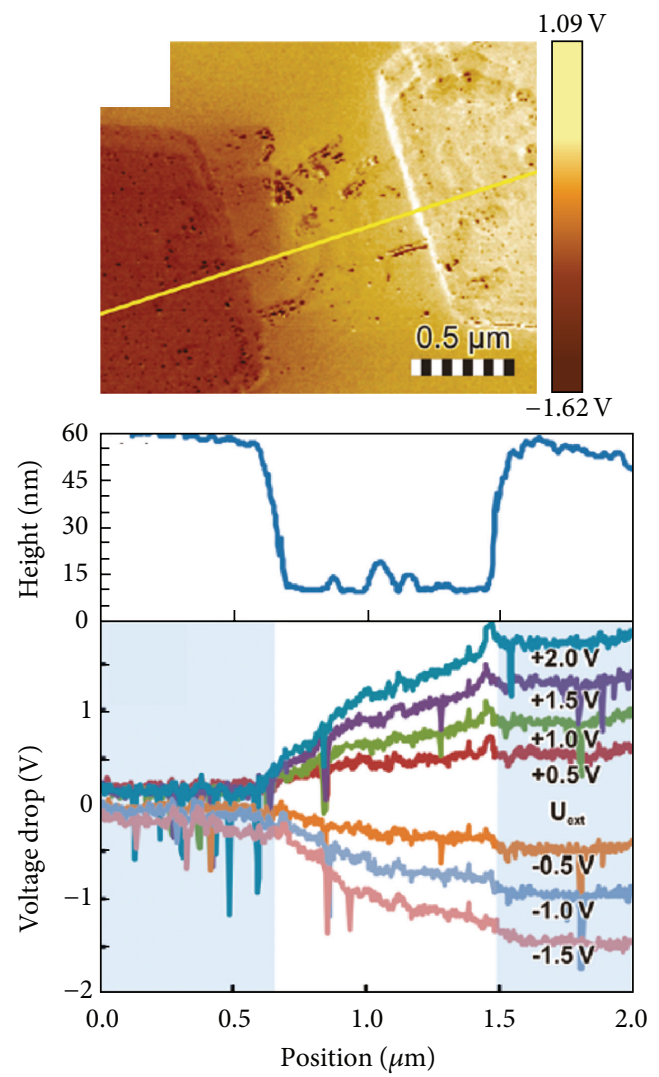

(b)

Figure 8: (a) The 2D and 3D surface topographs of a bare SiNW-FET device are shown. Figures reprinted with permission from [32], @ 2011 IOP Publishing Ltd. (b) Two-dimensional voltage drop image of the FGS at an external bias of +2 V. Figures reprinted with permission from [33], (C) 2011 ACS. 


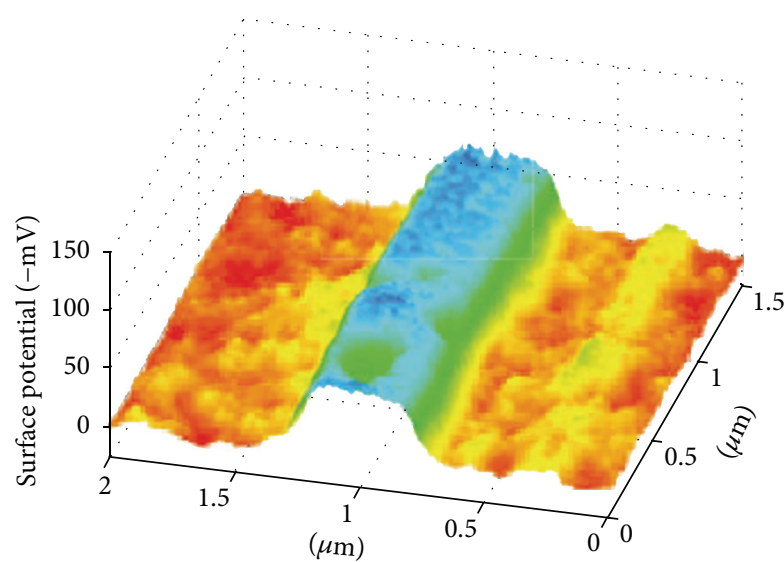

(a)

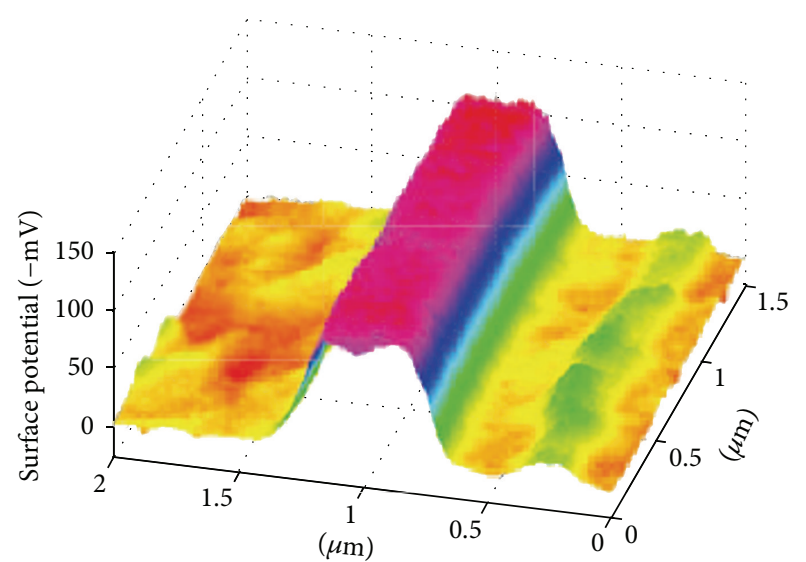

(b)

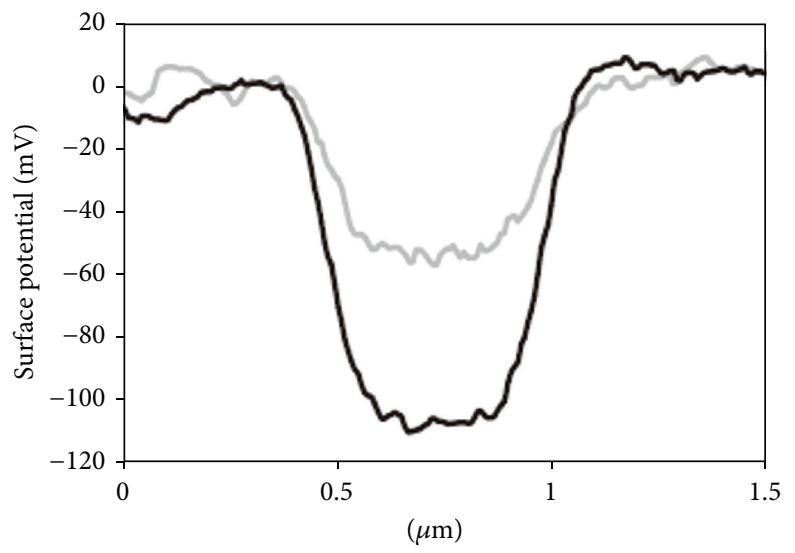

- Before complement

- After complement

(c)
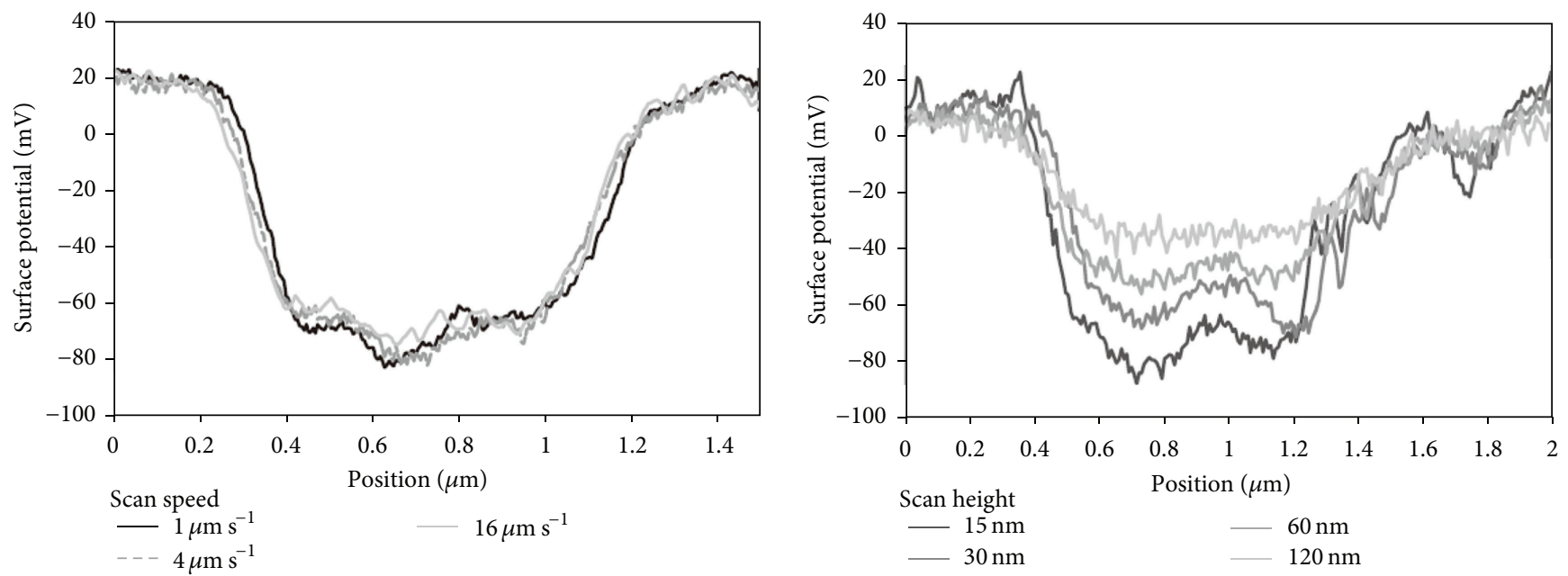

(d)

Complementary

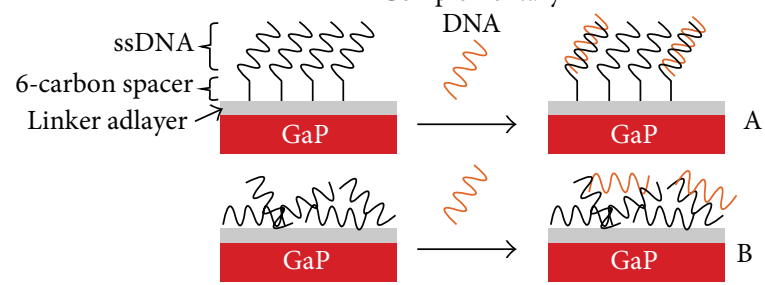

(e)

Figure 9: Continued. 


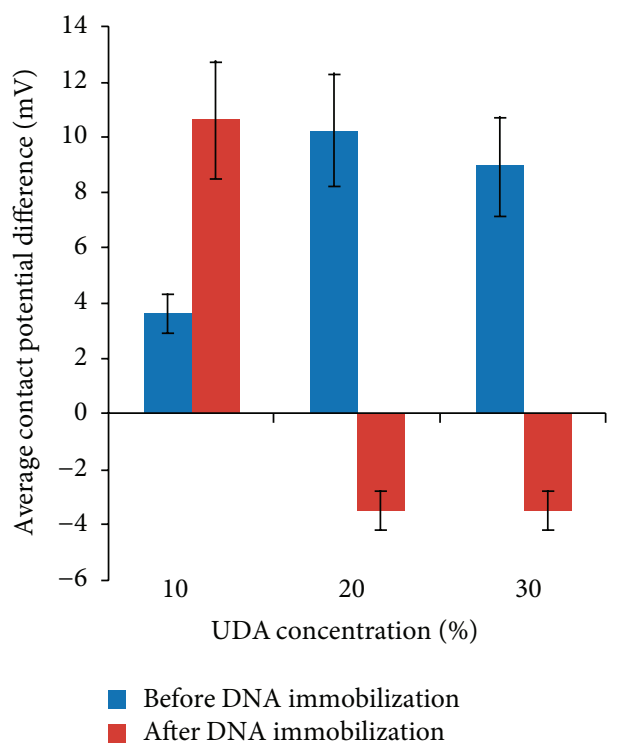

(f)

Figure 9: (a) The surface potential, measured with KPFM, of a patterned line of DNA probe strands taken from anthrax. (b) When a complementary target strand hybridizes with the anthrax probe, a clear signal doubling is observed. (c) Cross sections through the images in (a) and (b) make for an easy comparison of the surface potential before and after hybridization. (d) A quantitative look at the effect of scan speed and scan height on signal fidelity. Figures reprinted with permission from [34], ( 2007 NPG. (e) Two possible molecular arrangements of the DNA molecules on the GaP surface: (A) an ordered layer of DNA; (B) a more disordered layer of DNA. (f) Average contact potential difference $(\mathrm{mV})$ for various samples using 10, 20, and 30\% UDA concentration before and after DNA immobilization. Figures reprinted with permission from [35], (C) 2010 ACS.

above, it is obvious that KPFM retains a capacity for monitoring the conformational change of biomolecules during the immobilization or bioassay.

3.2.3. Sensing the Metal Ions Combined with Mismatched Nucleobases. KPFM technology can be also used to detect poisonous substances. Some metal ions including silver, mercury, and cadmium are the most dangerous ionic species for human health and ecosystems [101]. These hazardous species of metal pollutants widely contaminate ambient air, water, and soil, as a result of massive industrialization [102]. For sensing toxic metal ions, until the present, many researchers reported that nucleic acids and nucleobases specifically interacted with metal ions $[103,104]$. For instance, cytosine-cytosine (C-C) mismatch in DNA has the specificity for interacting with silver ions and it has attracted a great deal of attention with regard to metal ion sensing [105]. Thymine-thymine (T-T) mismatch can capture mercury ion and these mismatches are used in the detection protocol for sensing mercury ions [106]. Park and coworkers developed a highly sensitive detection method of silver ions, based on a combination of KPFM and DNA with desired sequence [36]. The topological and the surface potential images of both the DNA and the silver ion bound DNA were acquired by tapping mode AFM and KPFM, the results of which are shown in Figures 10(a)-10(d). The native DNA and the silver ion bound DNA have a similar height profiles in range of 3$5 \mathrm{~nm}$. However, they exhibited a large difference in surface potential $\left(\phi_{\mathrm{DNA}}=-88.34 \pm 6.01 \mathrm{mV}\right.$ and $\phi_{\mathrm{Ag}-\mathrm{DNA}}=-39.39 \pm$
$6.01 \mathrm{mV})$. Furthermore, the quantitative analysis of silver ion was successfully performed in the range of $0 \sim 2,000 \mathrm{fmol} \mathrm{Ag}^{+}$.

The detection protocol for highly sensitive detection of toxic mercury ion was also developed by Chanho and coworkers, based on a combination of KPFM and DNA with desired sequence [37]. The height of bare DNA was also measured to be $3 \sim 6 \mathrm{~nm}$ and there was no significant structural changes between the native DNA and the mercury ion bound DNA. However, they exhibited a large difference in surface potential $\left(\phi_{\mathrm{DNA}}=-150.8 \pm 5.26 \mathrm{mV}\right.$ and $\phi_{\mathrm{Hg}-\mathrm{DNA}}=$ $-116.69 \pm 4.64 \mathrm{mV})$ in Figures $10(\mathrm{e})-10(\mathrm{~h})$. The quantitative analysis of mercury ions was also performed in the range of $0 \sim 2,000 \mathrm{fmol}$. From this experiment, it was observed that the limit of detection of mercury ions was $\sim 2 \mathrm{fmol}$.

As describe above, a combination of KPFM and the DNA probe with specific sequence was used for ultrasensitive detection of silver and mercury ions. When this protocol was used, the sensitivity of the detection system was 1,000 times higher than that of the other methods. These results indicate that KPFM-based sensing platform has a great potential for providing a better performance into the detection of toxic ions such as silver and mercury.

3.2.4. Biomolecule Detection on a Patterned Carbon Nanotube. Patterned carbon nanotube (CNT) sensors have received a great amount of attention in bioanalytical science due to not only high specificity/sensitivity but also capability for quantitative analysis. Various methods including electrical and optical detections were utilized to monitor various 


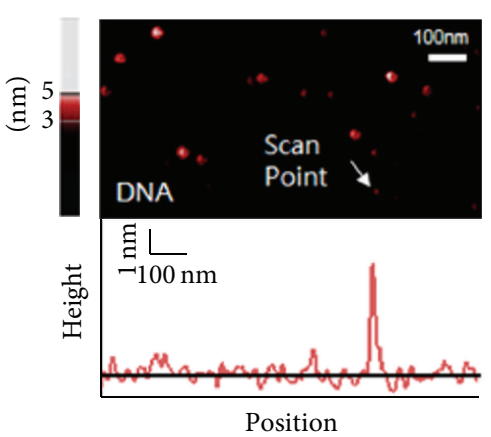

(a)

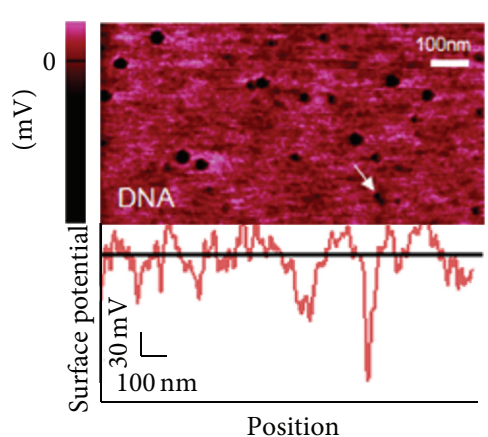

(c)
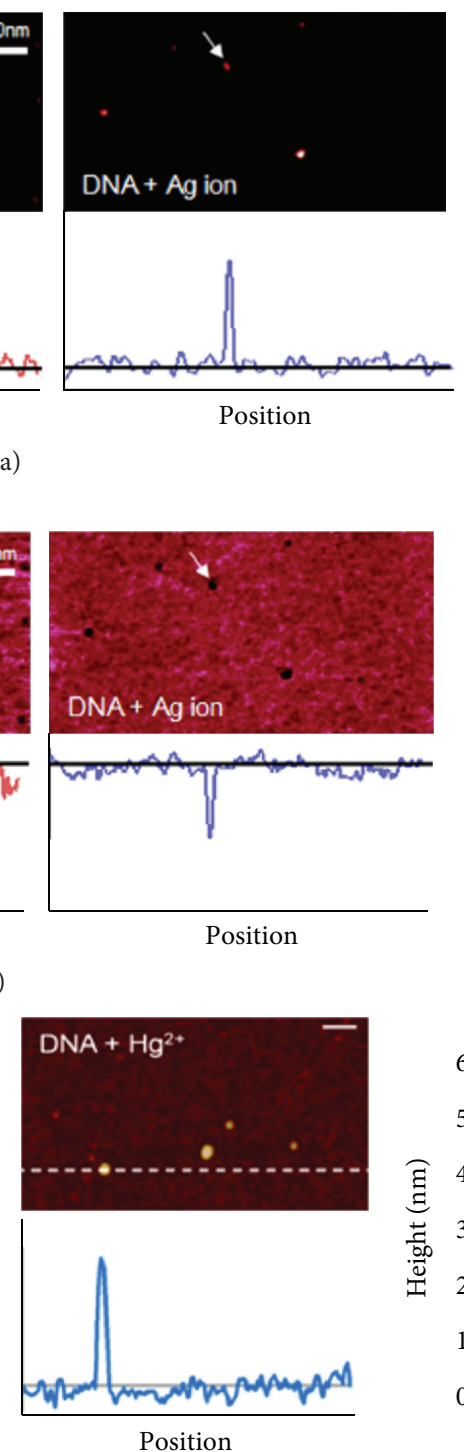

(e)

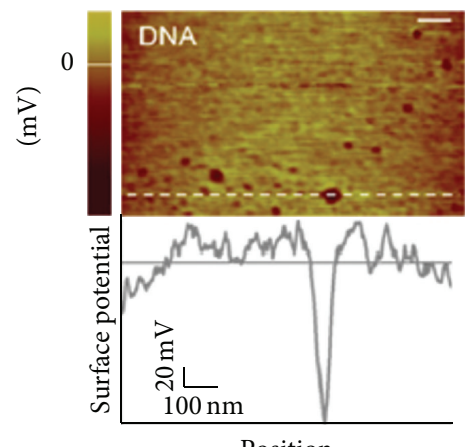

(g)

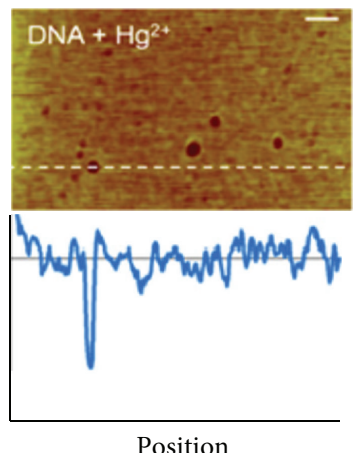

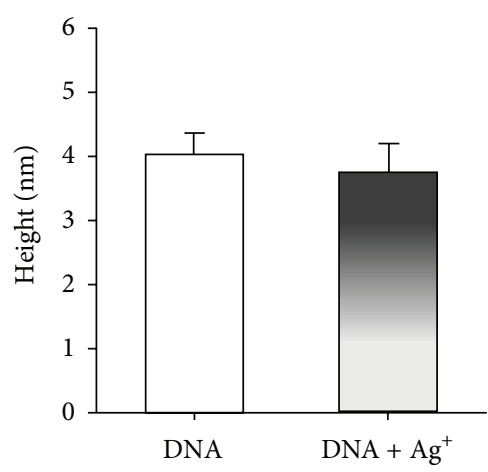

(b)

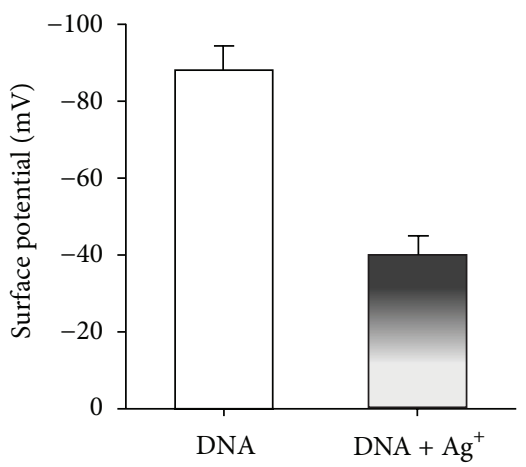

(d)

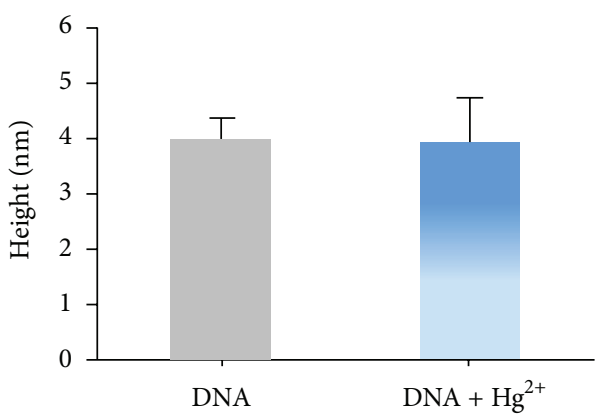

(f)

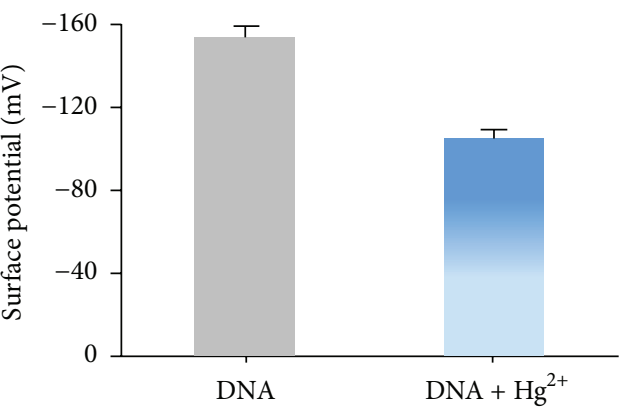

(h)

FIGURE 10: (a) Tapping mode AFM images of double strand DNA (marked as a white arrow) in unbound state (left) and silver ion bound state (right). (b) The average height of imaged double strand DNA in unbound state (white) and silver ion bound state (black). (c) KPFM images of double strand DNA (marked as a white arrow) in unbound state (left) and silver ion bound state (right). (d) Average surface potential of imaged double strand DNA in unbound state (white) and silver ion bound state (black). Figures reprinted with permission from [36], (c) 2014 Elsevier B.V. (e) Tapping mode AFM images of DNAs without $\mathrm{Hg}^{2+}$ (left) and $\mathrm{Hg}^{2+}$ bound state (right). (f) Average height of the measured bare DNA (grey) and $\mathrm{Hg}^{2+}$ bound DNA (blue). (g) KPFM images of DNA without $\mathrm{Hg}^{2+}$ (left) and $\mathrm{Hg}^{2+}$ bound state (right). The profiles below the images are the surface potential profiles corresponding to the white dash lines of the KPFM images. (h) Average surface potential of the bare DNA (grey) and $\mathrm{Hg}^{2+}$ bound DNA (blue). Figures reprinted with permission from [37], (c) 2015 IOP Publishing Ltd. 


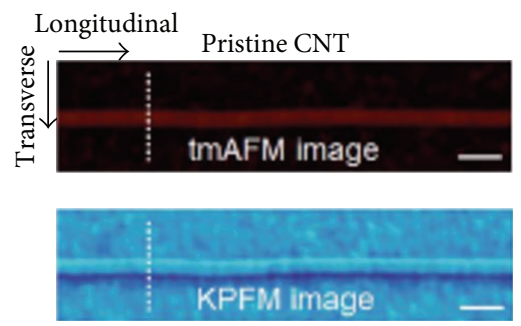

(a)
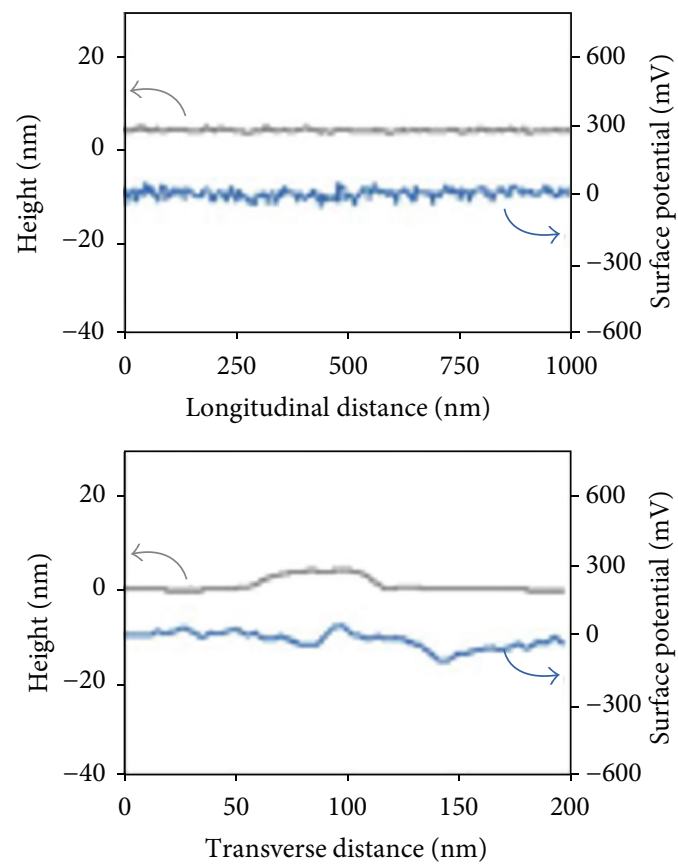

(d)

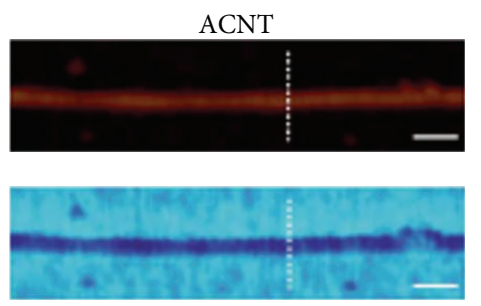

(b)

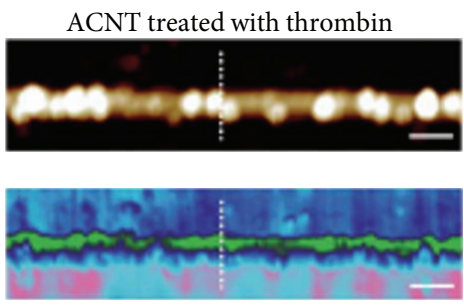

(c)
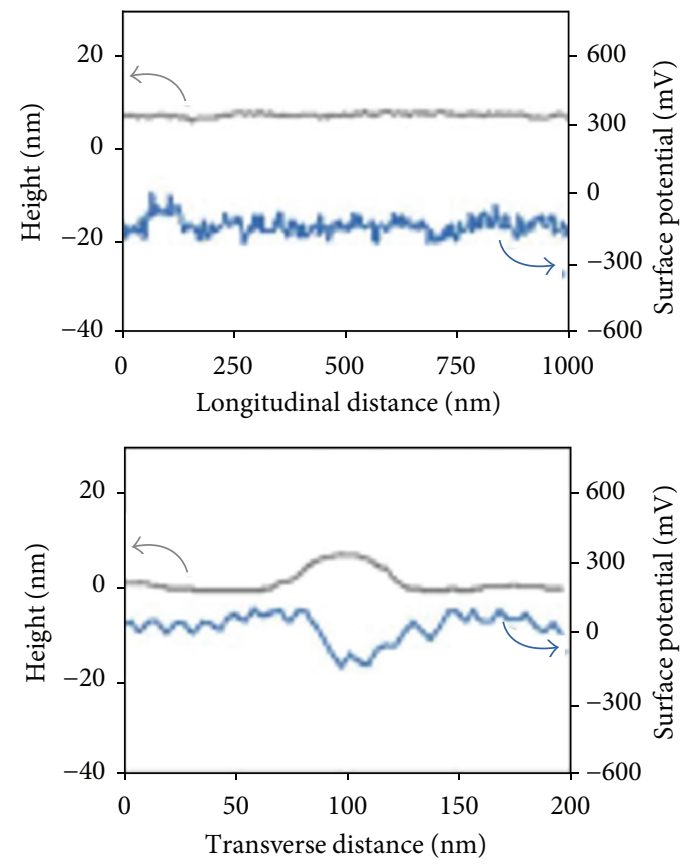

(e)
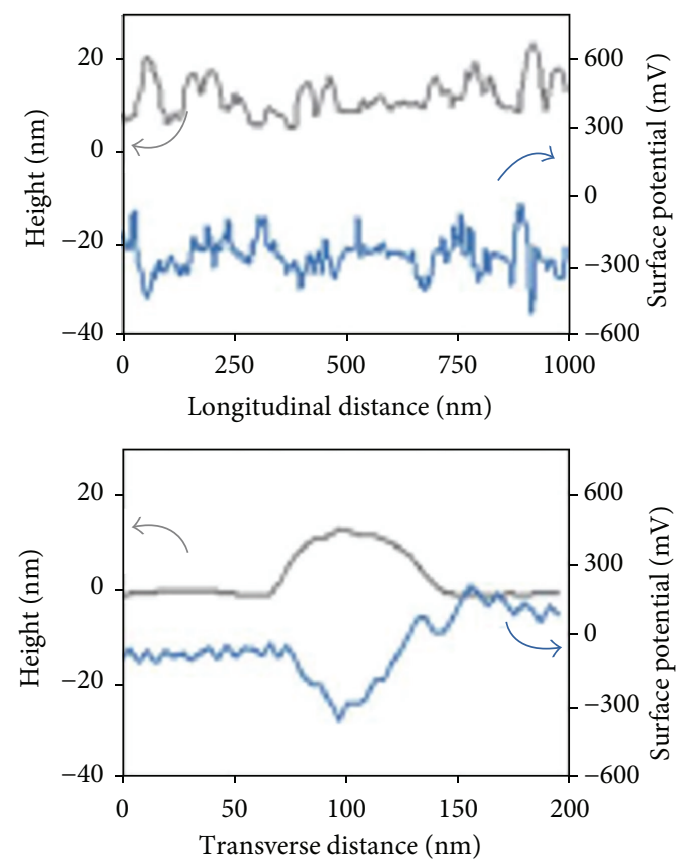

(f)

FIgure 11: Continued. 


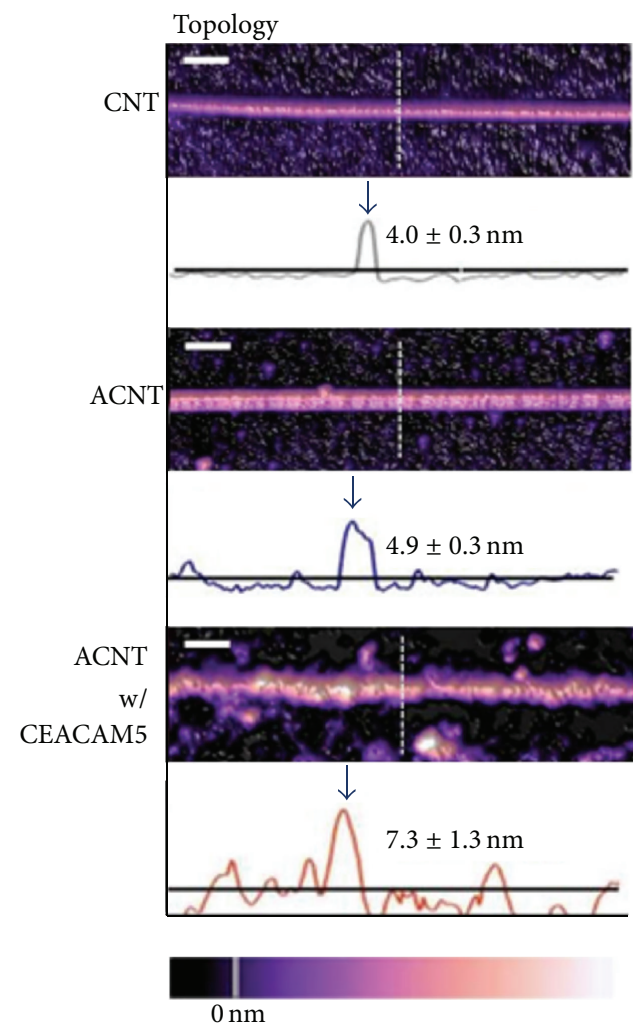

(g)

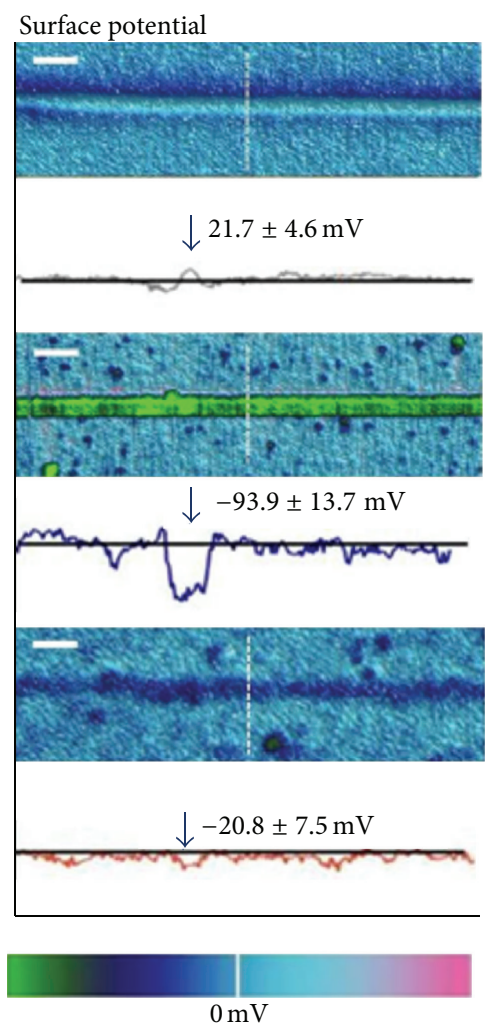

(h)

FIgURE 11: (a-c) Tapping mode AFM (upper panel) and KPFM (lower panel) images for pristine CNT, ACNT, and ACNT treated with thrombin. (d-f) The height and line potential profiles from tapping mode and KPFM images for the height and line potential obtained along the longitudinal (upper graph) and transverse (lower graph) directions. Scale bar is $100 \mathrm{~nm}$. Figures reprinted with permission from [25], (c) 2012 RSC. (g) AFM images and (h) KPFM images of a bare CNT, aptamer-functionalized CNT (ACNT), and aptamer-modified CNT capturing CEACAM5 molecules extracted from the LS174T cell lysate (1 cell/mL). Figures reprinted with permission from [38], (C) 2013 ACS.

biological interactions in aligned CNT biosensors. Recently, a new analytical method using KPFM has been proposed.

Nam and coworkers developed aligned CNT sensors having aptamers specific for thrombin and measured the surface potential variation of the sensors before and after thrombin addition [25]. Figures 11(a)-11(f) depict both the height and the surface potential images of bare CNT, aptamerconjugated CNT (ACNT), and thrombin-binding ACNT. The bare CNT was $4.4 \pm 1.5 \mathrm{~nm}$ in average height and the corresponding surface potential was $\sim 42.9 \mathrm{mV}$. Because of very low molecular weight of the aptamer $(19.6 \mathrm{Da})$, the height of ACNT was similar to that of the bare CNT. However, it exhibited a large difference in surface potential $\left(\phi_{\mathrm{ACNT}}=-142.5 \mathrm{mV}\right)$ due to negative charge of the backbone of aptamer. To verify whether the aptamer-conjugated CNTs are applicable to a biosensor, they measured the surface potential of the ACNTs which reacted with $1 \mathrm{nM}$ of thrombin molecules. The height profiles of the thrombin bound ACNTs were twice larger than that of the ACNT not treated with thrombin, and the surface potentials of them were more negative, exhibiting $\phi=\sim-263.8 \mathrm{mV}$. Furthermore, the KPFM quantitative analysis of thrombin was successfully performed using the ACNTs in the range of $1 \mathrm{pM} 1,000 \mathrm{pM}$ thrombin. In addition, it was observed that the interaction between the
ACNTs and the thrombins could be effectively inhibited by addition of antithrombin (10 10,000 pM).

Kwon and coworkers reported that a biosensor platform with the combination of ACNT and KPFM was also useful to perform the recognition of carcinoembryonic antigens (CEAs) expressed on circulating tumor cells (CTCs) [38]. They measured the surface potential differences of the ACNT based sensor before and after antigen treatment, as shown in Figures $11(\mathrm{~g})$ and $11(\mathrm{~h})$. The height of a bare CNT was $4.0 \pm 0.3 \mathrm{~nm}$ and the surface potential was measured to be $21.7 \pm 4.6 \mathrm{mV}$. The height of ACNT was similar to that of the bare CNT. However, it was shown that the surface potential of ACNT largely decreased, exhibiting $\phi=-93.9 \pm$ $13.7 \mathrm{mV}$. To conduct quantitative analysis of CEACAM5 proteins which bound to ACNT, the lysate solution of cancer cells reacted with the ACNT surface. When the CEACAM5 proteins bound to the ACNT surface, both the height and the surface potential of ACNT increased, exhibiting $h=$ $\sim 2.4 \mathrm{~nm}$ and $\phi=\sim 80 \mathrm{mV}$, respectively. Furthermore, the KPFM quantitative analysis of the CEACAM5 proteins was successfully performed in the range of $1 \sim 1,000$ cells/L. As described above, it is plausible that the combination of KPFM and ACNTs is a good platform for performing quantitative analysis of various biomolecules related to diseases. 
3.3. Perspective. As we have already discussed above, many researcher have reported various applications of KPFM ranging from material analysis to quantitative estimation of electrical properties of various devices [32, 94]. Because of KPFM's versatility for electrical measurement, KPFM could provide some important information in regard to distribution of surface potentials and surface potential densities of nanobiomaterials. Moreover, KPFM can also offer us some advantages to increase the effectiveness and performance of micro- and nanoscale devices. We also mentioned that KPFM has a great potential of being applicable to chemical or biological sensor systems [25-27]. In surface potential measurement, however, there are some limitations of KPFM including background noises from instrument or external defects, which affect surface potential images of a test sample, sometimes resulting in misleading measurement. To overcome these problems, KPFM technology has been upgraded by manufacturers, so that its spatial resolution, accuracy, and repeatability are improved. This progress will consistently take place in future. Another possibility to solve these limitations in applications of KPFM is utilization of nanoscale patterned substrates where nanomaterials such as nanoparticles, wires, and 2D sheets are aligned. Here, the nanopatterns act as templates in which biological interactions actively take place. Nanoscale patterned substrates allow us not only to increase sensitivity and specificity for detection of molecular interactions but also to precisely measure the surface potential without any artificial influence. Accordingly, we believe that if KPFM technology is consistently improved, KPFM can be used for a broader range of nanobioapplications in future.

\section{Conclusion}

As surveyed above, KPFM has been widely used to perform the surface characterization of various materials and devices due to its marvelous capabilities such as the surface potential measurement of the AuNPs, the accurate quantification of the electrical properties of graphene, and the electrical characterization of the $\mathrm{MoS}_{2}$ with various layers. That is because KPFM can provide researchers with a strong advantage of being able to measure the surface potentials of nanomaterials or devices at atomic/molecular scales. Moreover, it was proved that KPFM enabled monitoring the molecular interactions between biomolecules including the plasma membrane, the DNA hybridization, the ligand-protein kinase binding, and the aptamer-thrombin biding through surface potential measurement. Furthermore, several examples regarding quantitative bioanalysis using KPFM were systematically demonstrated. In other detection methods, monitoring of the abovementioned molecular interactions requires some labeling including fluorescent, electrochemical, or radioactive reagents. However, the KPFM based detection is basically label-free and requires no chemical reagents for improving image quality or contrast. Another advantage of KPFM is that it is able to effectively monitor the small ligand-protein binding that is important in signal transduction and onset of diseases. In general, the small ligand-protein binding can hardly be detected using other analytical methods, because the ligand is much smaller than the target proteins. Because of these advantages, KPFM is expected to attract much attention as an analytical tool in many research areas in future. In particular, it is considered that KPFM-based sensing technique can be used as a single-molecule diagnostic platform that may be useful for further applications including a precise prognosis of harmful diseases.

\section{Conflict of Interests}

The authors declare that there is no conflict of interests regarding the publication of this paper.

\section{Authors' Contribution}

Hyungbeen Lee and Wonseok Lee equally contributed to this work.

\section{Acknowledgment}

This work was supported by the National Research Foundation of Korea (NRF) Grant funded by the Korean Government (MSIP) (no. NRF-2015R1A5A7037674). J. H. Lee was supported by a Research Grant from Kwangwoon University in 2014.

\section{References}

[1] M. Nonnenmacher, M. P. O’Boyle, and H. K. Wickramasinghe, "Kelvin probe force microscopy," Applied Physics Letters, vol. 58, no. 25, pp. 2921-2923, 1991.

[2] W. Melitz, J. Shen, A. C. Kummel, and S. Lee, "Kelvin probe force microscopy and its application," Surface Science Reports, vol. 66, no. 1, pp. 1-27, 2011.

[3] A. Doukkali, S. Ledain, C. Guasch, and J. Bonnet, "Surface potential mapping of biased pn junction with kelvin probe force microscopy: application to cross-section devices," Applied Surface Science, vol. 235, no. 4, pp. 507-512, 2004.

[4] B. Moores, F. Hane, L. Eng, and Z. Leonenko, "Kelvin probe force microscopy in application to biomolecular films: frequency modulation, amplitude modulation, and lift mode," Ultramicroscopy, vol. 110, no. 6, pp. 708-711, 2010.

[5] K. Okamoto, Y. Sugawara, and S. Morita, "The imaging mechanism of atomic-scale Kelvin probe force microscopy and its application to atomic-scale force mapping," Japanese Journal of Applied Physics, vol. 42, no. 11, pp. 7163-7168, 2003.

[6] C. Barth, A. S. Foster, C. R. Henry, and A. L. Shluger, "Recent trends in surface characterization and chemistry with highresolution scanning force methods," Advanced Materials, vol. 23, no. 4, pp. 477-501, 2011.

[7] C. Sommerhalter, T. Glatzel, T. W. Matthes, A. Jäger-Waldau, and M. C. Lux-Steiner, "Kelvin probe force microscopy in ultra high vacuum using amplitude modulation detection of the electrostatic forces," Applied Surface Science, vol. 157, no. 4, pp. 263-268, 2000.

[8] O. Douhéret, A. Swinnen, S. Berthot et al., "High-resolution morphological and electrical characterisation of organic bulk heterojunction solar cells by scanning probe microscopy," Progress in Photovoltaics: Research and Applications, vol. 15, no. 8, pp. 713-726, 2007.

[9] O. Tal, W. Gao, C. K. Chan, A. Kahn, and Y. Rosenwaks, "Measurement of interface potential change and space charge region 
across metal/organic/metal structures using Kelvin probe force microscopy," Applied Physics Letters, vol. 85, no. 18, pp. 41484150, 2004.

[10] U. Zerweck, C. Loppacher, T. Otto, S. Grafström, and L. M. Eng, "Kelvin probe force microscopy of C60 on metal substrates: towards molecular resolution," Nanotechnology, vol. 18, no. 8, Article ID 084006, 2007.

[11] S. Lee, A. Shinde, and R. Ragan, "Morphological work function dependence of rare-earth disilicide metal nanostructures," Nanotechnology, vol. 20, no. 3, Article ID 035701, 2009.

[12] S. Sadewasser, P. Jelinek, C.-K. Fang et al., "New insights on atomic-resolution frequency-modulation Kelvin-probe forcemicroscopy imaging of semiconductors," Physical Review Letters, vol. 103, no. 26, Article ID 266103, 2009.

[13] S. Sadewasser, T. Glatzel, M. Rusu, A. Jäger-Waldau, and M. C. Lux-Steiner, "High-resolution work function imaging of single grains of semiconductor surfaces," Applied Physics Letters, vol. 80, no. 16, pp. 2979-2981, 2002.

[14] G. H. Buh, H. J. Chung, C. K. Kim, J. H. Yi, I. T. Yoon, and Y. Kuk, "Imaging of a silicon pn junction under applied bias with scanning capacitance microscopy and Kelvin probe force microscopy," Applied Physics Letters, vol. 77, no. 1, pp. 106-108, 2000.

[15] A. V. Ankudinov, V. P. Evtikhiev, K. S. Ladutenko, A. N. Titkov, and R. Laiho, "Kelvin probe force microscopy of hole leakage from the active region of a working injection-type semiconductor laser diode," Semiconductors, vol. 40, no. 8, pp. 982-989, 2006.

[16] P. Gao and Y. Cai, "Label-free detection of the aptamer binding on protein patterns using Kelvin probe force microscopy (KPFM)," Analytical and Bioanalytical Chemistry, vol. 394, no. 1, pp. 207-214, 2009.

[17] C. Leung, H. Kinns, B. W. Hoogenboom, S. Howorka, and P. Mesquida, "Imaging surface charges of individual biomolecules," Nano Letters, vol. 9, no. 7, pp. 2769-2773, 2009.

[18] C. Leung, D. Maradan, A. Kramer, S. Howorka, P. Mesquida, and B. W. Hoogenboom, "Improved Kelvin probe force microscopy for imaging individual DNA molecules on insulating surfaces," Applied Physics Letters, vol. 97, no. 20, Article ID 203703, 2010.

[19] G. Lee, W. Lee, H. Lee et al., "Mapping the surface charge distribution of amyloid fibril," Applied Physics Letters, vol. 101, no. 4, Article ID 043703, 2012.

[20] W. Lee, H. Jung, M. Son et al., "Characterization of the regrowth behavior of amyloid-like fragmented fibrils decomposed by ultrasonic treatment," RSC Advances, vol. 4, no. 100, pp. 5656156566, 2014.

[21] H. Schmidt, S. Habicht, S. Feste, A.-D. Müller, and O. G. Schmidt, "Kelvin probe force microscopy for characterizing doped semiconductors for future sensor applications in nanoand biotechnology," Applied Surface Science, vol. 281, pp. 24-29, 2013.

[22] L. Kelvin, "V. Contact electricity of metals," The London, Edinburgh, and Dublin Philosophical Magazine and Journal of Science, vol. 46, no. 278, pp. 82-120, 1898.

[23] V. Panchal, R. Pearce, R. Yakimova, A. Tzalenchuk, and O. Kazakova, "Standardization of surface potential measurements of graphene domains," Scientific Reports, vol. 3, article 2597, 2013.

[24] W. Lee, H. Lee, H. Jung et al., "Surface potential characterization of regrown amyloid fibrils originated from fragments by ultrasonic treatment," RSC Advances, vol. 4, no. 100, pp. 5656156566, 2014.

[25] K. Nam, K. Eom, J. Yang et al., "Aptamer-functionalized nanopattern based on carbon nanotube for sensitive, selective protein detection," Journal of Materials Chemistry, vol. 22, no. 44, pp. 23348-23356, 2012.

[26] J. Park, J. Yang, G. Lee et al., "Single-molecule recognition of biomolecular interaction via kelvin probe force microscopy," ACS Nano, vol. 5, no. 9, pp. 6981-6990, 2011.

[27] J. Park, D. Bang, K. Jang, S. Haam, J. Yang, and S. Na, “The work function of doped polyaniline nanoparticles observed by Kelvin probe force microscopy," Nanotechnology, vol. 23, no. 36, Article ID 365705, 2012.

[28] Y. Zhang, O. Pluchery, L. Caillard et al., "Sensing the charge state of single gold nanoparticles via work function measurements," Nano Letters, vol. 15, no. 1, pp. 51-55, 2015.

[29] O. Ochedowski, K. Marinov, N. Scheuschner et al., "Effect of contaminations and surface preparation on the work function of single layer $\mathrm{MoS}_{2}$," Beilstein Journal of Nanotechnology, vol. 5, no. 1, pp. 291-297, 2014.

[30] E. Drolle, R. M. Gaikwad, and Z. Leonenko, "Nanoscale electrostatic domains in cholesterol-laden lipid membranes create a target for amyloid binding," Biophysical Journal, vol. 103, no. 4, pp. L27-L29, 2012.

[31] Q. Ma, G. Wei, and X. Yang, "Influence of Au nanoparticles on the aggregation of amyloid- $\beta$-(25-35) peptides," Nanoscale, vol. 5, no. 21, pp. 10397-10403, 2013.

[32] C.-C. Tsai, P.-L. Chiang, C.-J. Sun et al., "Surface potential variations on a silicon nanowire transistor in biomolecular modification and detection," Nanotechnology, vol. 22, no. 13, Article ID 135503, 2011.

[33] L. Yan, C. Punckt, I. A. Aksay, W. Mertin, and G. Bacher, "Local voltage drop in a single functionalized graphene sheet characterized by Kelvin probe force microscopy," Nano Letters, vol. 11, no. 9, pp. 3543-3549, 2011.

[34] A. K. Sinensky and A. M. Belcher, "Label-free and high-resolution protein/DNA nanoarray analysis using Kelvin probe force microscopy," Nature Nanotechnology, vol. 2, no. 10, pp. 653-659, 2007.

[35] D. N. Richards, D. Y. Zemlyanov, R. M. Asrar et al., "DNA immobilization on $\mathrm{GaP}(100)$ investigated by kelvin probe force microscopy," The Journal of Physical Chemistry C, vol. 114, no. 36, pp. 15486-15490, 2010.

[36] J. Park, S. Lee, K. Jang, and S. Na, "Ultra-sensitive direct detection of silver ions via Kelvin probe force microscopy," Biosensors and Bioelectronics, vol. 60, pp. 299-304, 2014.

[37] P. Chanho, J. Kuewhan, L. Sangmyung et al., "A highly sensitive, direct and label-free technique for $\mathrm{Hg}^{2+}$ detection using Kelvin probe force microscopy," Nanotechnology, vol. 26, no. 30, Article ID 305501, 2015.

[38] T. Kwon, J. Park, G. Lee et al., "Carbon nanotube-patterned surface-based recognition of carcinoembryonic antigens in tumor cells for cancer diagnosis," The Journal of Physical Chemistry Letters, vol. 4, no. 7, pp. 1126-1130, 2013.

[39] J. H. Choi, F. T. Nguyen, P. W. Barone et al., "Multimodal biomedical imaging with asymmetric single-walled carbon nanotube/iron oxide nanoparticle complexes," Nano Letters, vol. 7, no. 4, pp. 861-867, 2007.

[40] J. R. McCarthy, F. A. Jaffer, and R. Weissleder, "A macrophagetargeted theranostic nanoparticle for biomedical applications," Small, vol. 2, no. 8-9, pp. 983-987, 2006. 
[41] S. O. Kelley, C. A. Mirkin, D. R. Walt, R. F. Ismagilov, M. Toner, and E. H. Sargent, "Advancing the speed, sensitivity and accuracy of biomolecular detection using multi-length-scale engineering," Nature Nanotechnology, vol. 9, no. 12, pp. 969-980, 2014.

[42] A. N. Shipway, E. Katz, and I. Willner, "Nanoparticle arrays on surfaces for electronic, optical, and sensor applications," ChemPhysChem, vol. 1, no. 1, pp. 18-52, 2000.

[43] A. D. McFarland and R. P. Van Duyne, "Single silver nanoparticles as real-time optical sensors with zeptomole sensitivity," Nano Letters, vol. 3, no. 8, pp. 1057-1062, 2003.

[44] S. Oldenburg, R. Averitt, S. Westcott, and N. Halas, "Nanoengineering of optical resonances," Chemical Physics Letters, vol. 288, no. 2, pp. 243-247, 1998.

[45] F. Verbakel, S. C. J. Meskers, and R. A. J. Janssen, "Electronic memory effects in diodes from a zinc oxide nanoparticle-polystyrene hybrid material," Applied Physics Letters, vol. 89, no. 10, Article ID 102103, 2006.

[46] M.-C. Daniel and D. Astruc, "Gold nanoparticles: assembly, supramolecular chemistry, quantum-size-related properties, and applications toward biology, catalysis, and nanotechnology," Chemical Reviews, vol. 104, no. 1, pp. 293-346, 2004.

[47] Y.-C. Chuang, J.-C. Li, S.-H. Chen et al., "An optical biosensing platform for proteinase activity using gold nanoparticles," Biomaterials, vol. 31, no. 23, pp. 6087-6095, 2010.

[48] B. K. Jena and C. R. Raj, "Optical sensing of biomedically important polyionic drugs using nano-sized gold particles," Biosensors and Bioelectronics, vol. 23, no. 8, pp. 1285-1290, 2008.

[49] C. Gutiérrez-Sánchez, M. Pita, C. Vaz-Domínguez, S. Shleev, and A. L. De Lacey, "Gold nanoparticles as electronic bridges for laccase-based biocathodes," Journal of the American Chemical Society, vol. 134, no. 41, pp. 17212-17220, 2012.

[50] G. Raschke, S. Kowarik, T. Franzl et al., "Biomolecular recognition based on single gold nanoparticle light scattering," Nano Letters, vol. 3, no. 7, pp. 935-938, 2003.

[51] Y.-C. Lin, B.-Y. Yu, W.-C. Lin, S.-H. Lee, C.-H. Kuo, and J.J. Shyue, "Tailoring the surface potential of gold nanoparticles with self-assembled monolayers with mixed functional groups," Journal of Colloid and Interface Science, vol. 340, no. 1, pp. 126130, 2009.

[52] D. Conklin, S. Nanayakkara, T.-H. Park et al., "Electronic transport in porphyrin supermolecule-gold nanoparticle assemblies," Nano Letters, vol. 12, no. 5, pp. 2414-2419, 2012.

[53] S. Stehlik, T. Petit, H. A. Girard, A. Kromka, J.-C. Arnault, and B. Rezek, "Surface potential of diamond and gold nanoparticles can be locally switched by surrounding materials or applied voltage," Journal of Nanoparticle Research, vol. 16, no. 4, pp. 1-11, 2014.

[54] K. S. Novoselov, D. Jiang, F. Schedin et al., "Two-dimensional atomic crystals," Proceedings of the National Academy of Sciences of the United States of America, vol. 102, no. 30, pp. 10451-10453, 2005.

[55] Q. H. Wang, K. Kalantar-Zadeh, A. Kis, J. N. Coleman, and M. S. Strano, "Electronics and optoelectronics of two-dimensional transition metal dichalcogenides," Nature Nanotechnology, vol. 7, no. 11, pp. 699-712, 2012.

[56] A. K. Geim and K. S. Novoselov, "The rise of graphene," Nature Materials, vol. 6, no. 3, pp. 183-191, 2007.

[57] C. N. R. Rao, A. K. Sood, K. S. Subrahmanyam, and A. Govindaraj, "Graphene: the new two-dimensional nanomaterial," Angewandte Chemie - International Edition, vol. 48, no. 42, pp. 7752-7777, 2009.
[58] A. K. Geim, “Graphene: status and prospects," Science, vol. 324, no. 5934, pp. 1530-1534, 2009.

[59] R. Balog, B. Jørgensen, L. Nilsson et al., "Bandgap opening in graphene induced by patterned hydrogen adsorption," Nature Materials, vol. 9, no. 4, pp. 315-319, 2010.

[60] B. Radisavljevic, A. Radenovic, J. Brivio, V. Giacometti, and A. Kis, "Single-layer $\mathrm{MoS}_{2}$ transistors," Nature Nanotechnology, vol. 6, no. 3, pp. 147-150, 2011.

[61] A. Splendiani, L. Sun, Y. Zhang et al., "Emerging photoluminescence in monolayer MoS2," Nano Letters, vol. 10, no. 4, pp. 1271-1275, 2010.

[62] J. Kwon, Y. K. Hong, G. Han et al., "Giant photoamplification in indirect-bandgap multilayer MoS2 phototransistors with local bottom-gate structures," Advanced Materials, vol. 27, no. 13, pp. 2224-2230, 2015.

[63] C. Lee, H. Yan, L. E. Brus, T. F. Heinz, J. Hone, and S. Ryu, "Anomalous lattice vibrations of single-and few-layer $\mathrm{MoS}_{2}$," ACS Nano, vol. 4, no. 5, pp. 2695-2700, 2010.

[64] H. S. Lee, S.-W. Min, Y.-G. Chang et al., " $\mathrm{MoS}_{2}$ nanosheet phototransistors with thickness-modulated optical energy gap," Nano Letters, vol. 12, no. 7, pp. 3695-3700, 2012.

[65] E. Finote, Y. Leonenko, B. Moores, L. Eng, M. Amrein, and Z. Leonenko, "Effect of cholesterol on electrostatics in lipid-protein films of a pulmonary surfactant," Langmuir, vol. 26, no. 3, pp. 1929-1935, 2010.

[66] P. Zhang and H. F. Cantiello, "Measurement of charge distribution in actin bundles by surface potential microscopy," Applied Physics Letters, vol. 95, no. 3, Article ID 033701, 2009.

[67] P. Zhang and H. F. Cantiello, "Electrical mapping of microtubular structures by surface potential microscopy," Applied Physics Letters, vol. 95, no. 11, Article ID 113703, 2009.

[68] S. Hayakawa and S. Nakai, "Relationships of hydrophobicity and net charge to the solubility of milk and soy proteins," Journal of Food Science, vol. 50, no. 2, pp. 486-491, 1985.

[69] S. Koch, P. Woias, L. K. Meixner, S. Drost, and H. Wolf, "Protein detection with a novel ISFET-based zeta potential analyzer," Biosensors and Bioelectronics, vol. 14, no. 4, pp. 413-421, 1999.

[70] D. Möckel, E. Staude, M. Dal-Cin, K. Darcovich, and M. Guiver, "Tangential flow streaming potential measurements: hydrodynamic cell characterization and zeta potentials of carboxylated polysulfone membranes," Journal of Membrane Science, vol. 145, no. 2, pp. 211-222, 1998.

[71] H. F. Knapp, P. Mesquida, and A. Stemmer, "Imaging the surface potential of active purple membrane," Surface and Interface Analysis, vol. 33, no. 2, pp. 108-112, 2002.

[72] X. G. Briones, M. D. Urzúa, H. E. Ríos, F. J. Espinoza-Beltrán, R. Dabirian, and M. Yazdani-Pedram, "Thin films of amphiphilic polyelectrolytes. Soft materials characterized by Kelvin probe force microscopy," Polymer, vol. 54, no. 21, pp. 5733-5740, 2013.

[73] M. E. Davis and J. A. McCammon, "Electrostatics in biomolecular structure and dynamics," Chemical Reviews, vol. 90, no. 3, pp. 509-521, 1990.

[74] M. J. Packer, M. P. Dauncey, and C. A. Hunter, "Sequencedependent DNA structure: dinucleotide conformational maps," Journal of Molecular Biology, vol. 295, no. 1, pp. 71-83, 2000.

[75] C. M. Dobson, "Protein folding and misfolding," Nature, vol. 426, no. 6968, pp. 884-890, 2003.

[76] D. J. Selkoe, "Folding proteins in fatal ways," Nature, vol. 426, no. 6968, pp. 900-904, 2003.

[77] G. Merlini and V. Bellotti, "Molecular mechanisms of amyloidosis," The New England Journal of Medicine, vol. 349, no. 6, pp. 583-596, 2003. 
[78] S. D. Ginsberg, J. E. Galvin, T.-S. Chiu, V. M.-Y. Lee, E. Masliah, and J. Q. Trojanowski, "RNA sequestration to pathological lesions of neurodegenerative diseases," Acta Neuropathologica, vol. 96, no. 5, pp. 487-494, 1998.

[79] N. R. Deleault, R. W. Lucassen, and S. Supattapone, "RNA molecules stimulate prion protein conversion," Nature, vol. 425, no. 6959, pp. 717-720, 2003.

[80] Y. Verdier, M. Zarándi, and B. Penke, "Amyloid $\beta$-peptide interactions with neuronal and glial cell plasma membrane: binding sites and implications for Alzheimer's disease," Journal of Peptide Science, vol. 10, no. 5, pp. 229-248, 2004.

[81] C. Li, J. Adamcik, and R. Mezzenga, "Biodegradable nanocomposites of amyloid fibrils and graphene with shape-memory and enzyme-sensing properties," Nature Nanotechnology, vol. 7, no. 7, pp. 421-427, 2012.

[82] C. Li, S. Bolisetty, and R. Mezzenga, "Hybrid nanocomposites of gold single-crystal platelets and amyloid fibrils with tunable fluorescence, conductivity, and sensing properties," Advanced Materials, vol. 25, no. 27, pp. 3694-3700, 2013.

[83] C. Li and R. Mezzenga, "The interplay between carbon nanomaterials and amyloid fibrils in bio-nanotechnology," Nanoscale, vol. 5, no. 14, pp. 6207-6218, 2013.

[84] J. Adamcik, J.-M. Jung, J. Flakowski, P. De Los Rios, G. Dietler, and R. Mezzenga, "Understanding amyloid aggregation by statistical analysis of atomic force microscopy images," Nature Nanotechnology, vol. 5, no. 6, pp. 423-428, 2010.

[85] J.-M. Jung, G. Savin, M. Pouzot, C. Schmitt, and R. Mezzenga, "Structure of heat-induced $\beta$-lactoglobulin aggregates and their complexes with sodium-dodecyl sulfate," Biomacromolecules, vol. 9, no. 9, pp. 2477-2486, 2008.

[86] J.-P. Knemeyer, N. Marmé, and M. Sauer, "Probes for detection of specific DNA sequences at the single-molecule level," Analytical Chemistry, vol. 72, no. 16, pp. 3717-3724, 2000.

[87] Y. Yang, Y. Xu, T. Xia et al., "A single-molecule study of the inhibition effect of Naringenin on transforming growth factor$\beta$ ligand-receptor binding," Chemical Communications, vol. 47, no. 19, pp. 5440-5442, 2011.

[88] C. Stroh, H. Wang, R. Bash et al., "Single-molecule recognition imaging microscopy," Proceedings of the National Academy of Sciences of the United States of America, vol. 101, no. 34, pp. 12503-12507, 2004.

[89] S. Allen, X. Chen, J. Davies et al., "Detection of antigenantibody binding events with the atomic force microscope," Biochemistry, vol. 36, no. 24, pp. 7457-7463, 1997.

[90] D. N. Richards, D. Y. Zemlyanov, and A. Ivanisevic, "Kelvin probe force microscopy analysis of the covalent functionalization and DNA modification of gallium phosphide nanorods," The Journal of Physical Chemistry C, vol. 116, no. 23, pp. 1261312620, 2012.

[91] B. Moores, J. Simons, S. Xu, and Z. Leonenko, "AFM-assisted fabrication of thiol SAM pattern with alternating quantified surface potential," Nanoscale Research Letters, vol. 6, no. 1, pp. $1-5,2011$.

[92] C.-C. Tsai, H.-H. Hung, C.-P. Liu, Y.-T. Chen, and C.-Y. Pan, "Changes in plasma membrane surface potential of PC12 cells as measured by Kelvin probe force microscopy," PLoS ONE, vol. 7, no. 4, Article ID e33849, 2012.

[93] A. Liscio, G. P. Veronese, E. Treossi et al., "Charge transport in graphene-polythiophene blends as studied by Kelvin Probe Force Microscopy and transistor characterization," Journal of Materials Chemistry, vol. 21, no. 9, pp. 2924-2931, 2011.
[94] D. Luo, H. Sun, and Y. Li, "Kelvin probe force microscopy in nanoscience and nanotechnology," in Surface Science Tools for Nanomaterials Characterization, pp. 117-158, Spriger, Berlin, Germany, 2015.

[95] D. Bitounis, H. Ali-Boucetta, B. H. Hong, D.-H. Min, and K. Kostarelos, "Prospects and challenges of graphene in biomedical applications," Advanced Materials, vol. 25, no. 16, pp. 22582268, 2013

[96] K. S. Novoselov, V. I. Fal'Ko, L. Colombo, P. R. Gellert, M. G. Schwab, and K. Kim, "A roadmap for graphene," Nature, vol. 490, no. 7419, pp. 192-200, 2012.

[97] E. Mikamo-Satoh, F. Yamada, A. Takagi, T. Matsumoto, and T. Kawai, "Electrostatic force microscopy: imaging DNA and protein polarizations one by one," Nanotechnology, vol. 20, no. 14, Article ID 145102, 2009.

[98] M. Delalande, S. Clavaguera, M. Toure et al., "Chemical functionalization of electrodes for detection of gaseous nerve agents with carbon nanotube field-effect transistors," Chemical Communications, vol. 47, no. 21, pp. 6048-6050, 2011.

[99] N. Shirahata, S. Furumi, Y. Masuda, A. Hozumi, and Y. Sakka, "Fluorescence detection and imaging of amino-functionalized organic monolayer," Thin Solid Films, vol. 516, no. 9, pp. 25412546, 2008.

[100] L. Frolov, Y. Rosenwaks, S. Richter, C. Carmeli, and I. Carmeli, "Photoelectric junctions between GaAs and photosynthetic reaction center protein," The Journal of Physical Chemistry C, vol. 112, no. 35, pp. 13426-13430, 2008.

[101] D. Faye, H. Zhang, J.-P. Lefevre, J. Bell, J. A. Delaire, and I. Leray, "Mercury detection in a microfluidic device by using a molecular sensor soluble in organoaqueous solvent," Photochemical \& Photobiological Sciences, vol. 11, no. 11, pp. 1737-1743, 2012.

[102] C. M. Wood, M. D. McDonald, P. Walker et al., "Bioavailability of silver and its relationship to ionoregulation and silver speciation across a range of salinities in the gulf toadfish (Opsanus beta)," Aquatic Toxicology, vol. 70, no. 2, pp. 137-157, 2004.

[103] G. H. Clever, C. Kaul, and T. Carell, "DNA-metal base pairs," Angewandte Chemie - International Edition, vol. 46, no. 33, pp. 6226-6236, 2007.

[104] Y. Miyake, H. Togashi, M. Tashiro et al., "MercuryII-mediated formation of thymine-HgII-thymine base pairs in DNA duplexes," Journal of the American Chemical Society, vol. 128, no. 7, pp. 2172-2173, 2006.

[105] H. Gong and X. Li, "Y-type, C-rich DNA probe for electrochemical detection of silver ion and cysteine," Analyst, vol. 136, no. 11, pp. 2242-2246, 2011.

[106] S. Wen, T. Zeng, L. Liu et al., "Highly sensitive and selective DNA-based detection of mercury(II) with $\alpha$-hemolysin nanopore," Journal of the American Chemical Society, vol. 133, no. 45, pp. 18312-18317, 2011. 

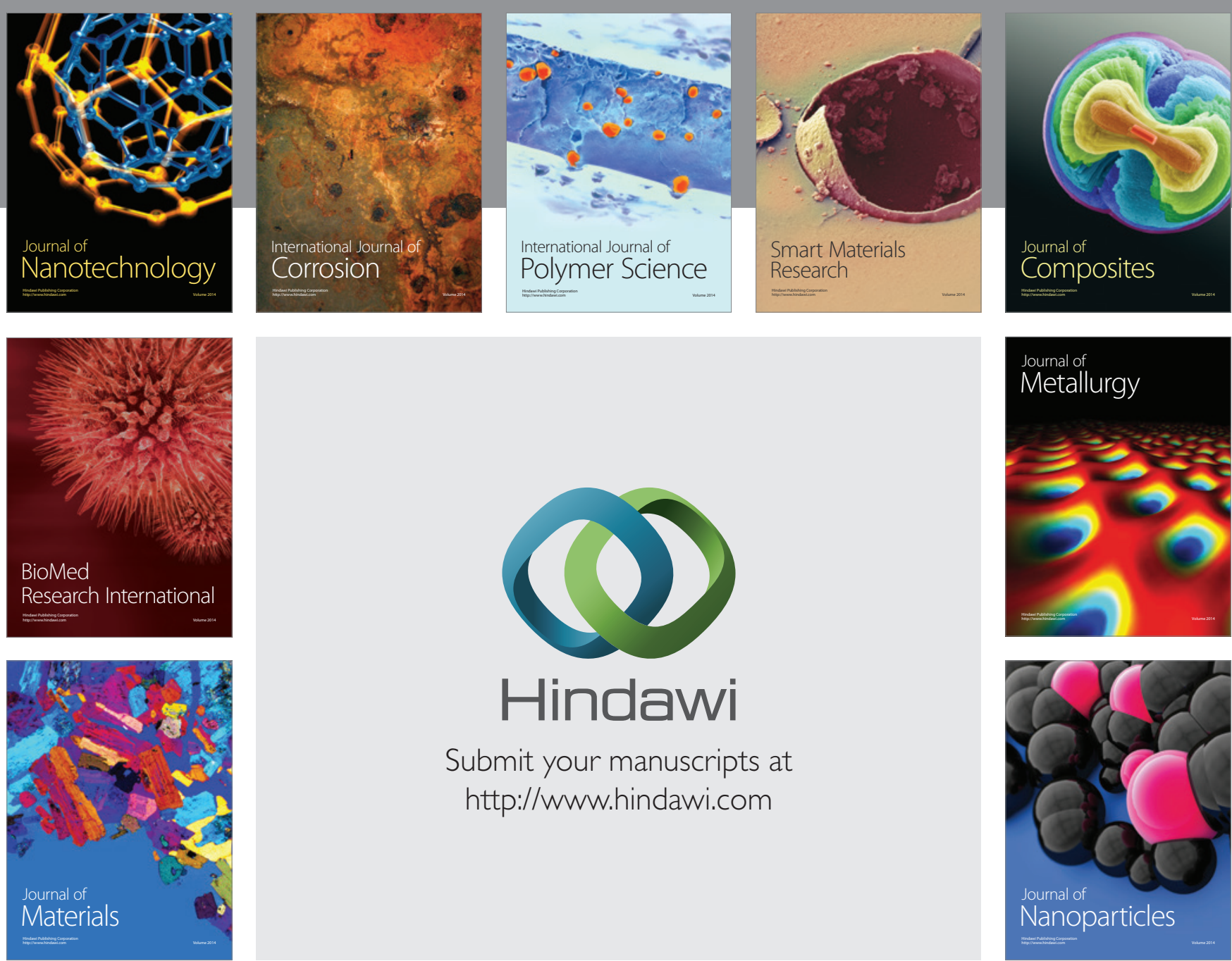

\section{Hindawi}

Submit your manuscripts at

http://www.hindawi.com

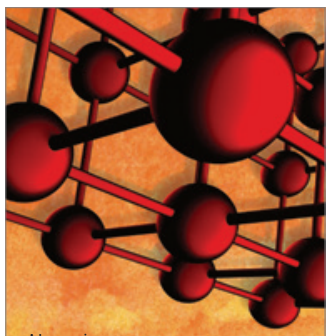

Materials Science and Engineering
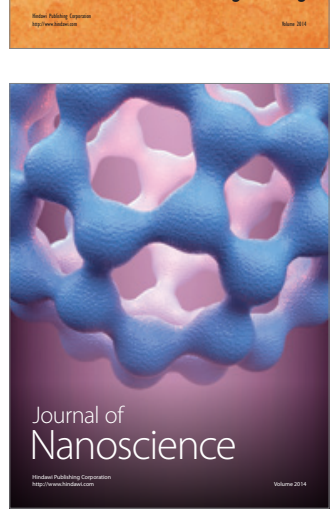
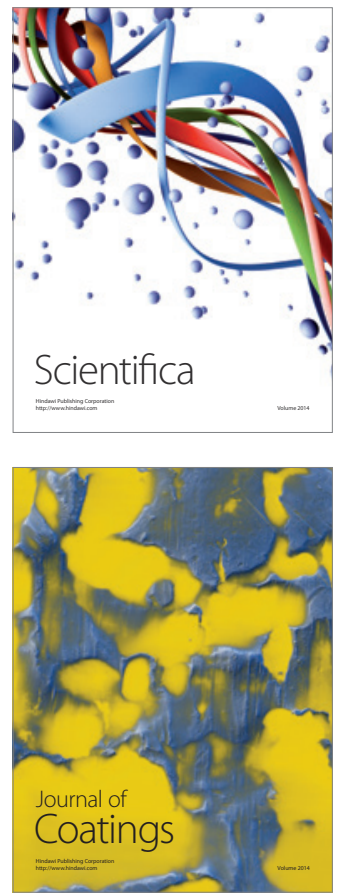
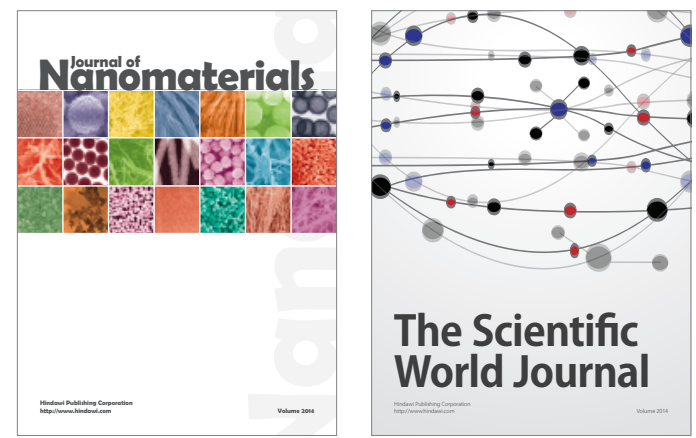

The Scientific World Journal
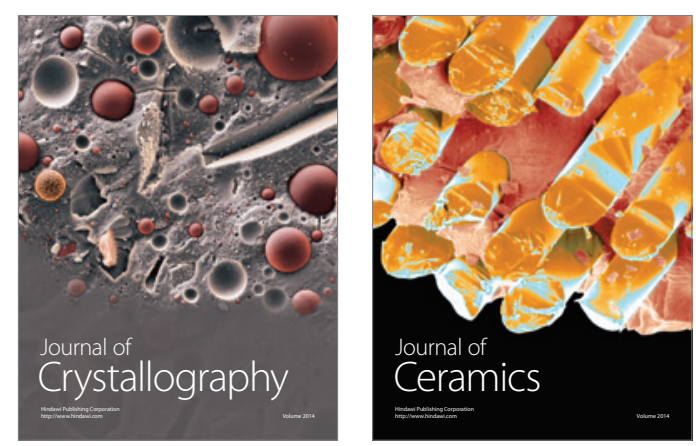
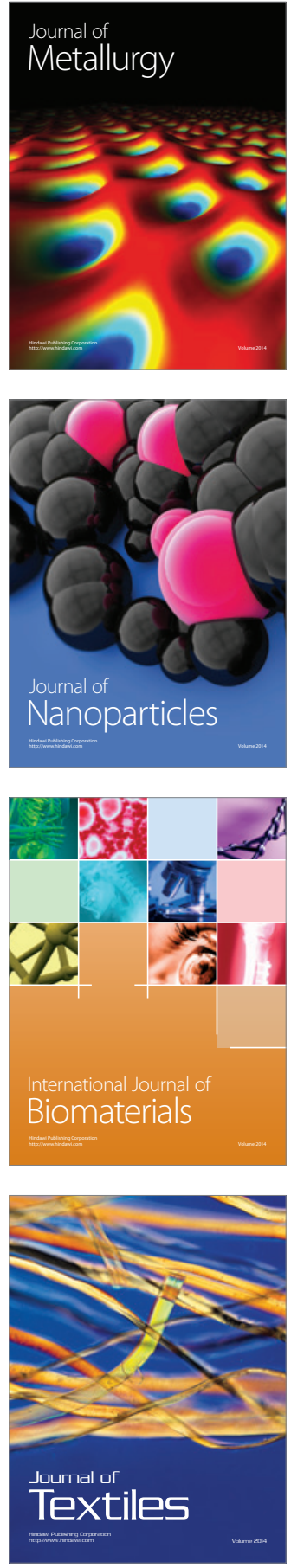